NASA/CR-2005-213034

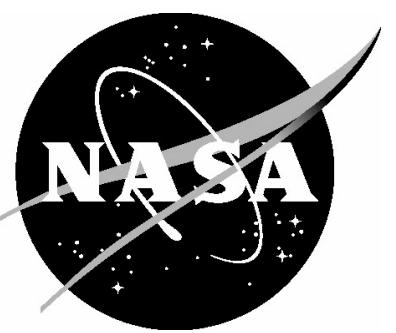

\title{
Conceptual Design of a Communications Relay Satellite for a Lunar Sample Return Mission
}

Christopher W. Brunner

The George Washington University

Joint Institute for Advancement of Flight Sciences

Langley Research Center, Hampton, Virginia 
Since its founding, NASA has been dedicated to the advancement of aeronautics and space science. The NASA Scientific and Technical Information (STI) Program Office plays a key part in helping NASA maintain this important role.

The NASA STI Program Office is operated by Langley Research Center, the lead center for NASA's scientific and technical information. The NASA STI Program Office provides access to the NASA STI Database, the largest collection of aeronautical and space science STI in the world. The Program Office is also NASA's institutional mechanism for disseminating the results of its research and development activities. These results are published by NASA in the NASA STI Report Series, which includes the following report types:

- TECHNICAL PUBLICATION. Reports of completed research or a major significant phase of research that present the results of NASA programs and include extensive data or theoretical analysis. Includes compilations of significant scientific and technical data and information deemed to be of continuing reference value. NASA counterpart of peerreviewed formal professional papers, but having less stringent limitations on manuscript length and extent of graphic presentations.

- TECHNICAL MEMORANDUM. Scientific and technical findings that are preliminary or of specialized interest, e.g., quick release reports, working papers, and bibliographies that contain minimal annotation. Does not contain extensive analysis.

- CONTRACTOR REPORT. Scientific and technical findings by NASA-sponsored contractors and grantees.
- CONFERENCE PUBLICATION. Collected papers from scientific and technical conferences, symposia, seminars, or other meetings sponsored or co-sponsored by NASA.

- SPECIAL PUBLICATION. Scientific, technical, or historical information from NASA programs, projects, and missions, often concerned with subjects having substantial public interest.

- TECHNICAL TRANSLATION. Englishlanguage translations of foreign scientific and technical material pertinent to NASA's mission.

Specialized services that complement the STI Program Office's diverse offerings include creating custom thesauri, building customized databases, organizing and publishing research results ... even providing videos.

For more information about the NASA STI Program Office, see the following:

- Access the NASA STI Program Home Page at http://www.sti.nasa.gov

- E-mail your question via the Internet to help@sti.nasa.gov

- Fax your question to the NASA STI Help Desk at (301) 621-0134

- Phone the NASA STI Help Desk at (301) 621-0390

- Write to:

NASA STI Help Desk NASA Center for AeroSpace Information 7121 Standard Drive Hanover, MD 21076-1320 
NASA/CR-2005-213034

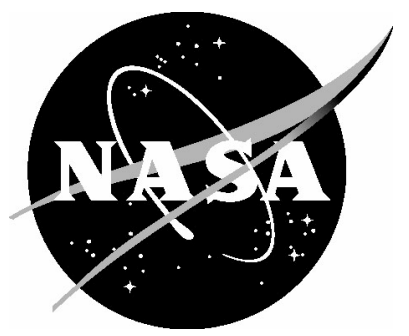

\section{Conceptual Design of a Communications Relay Satellite for a Lunar Sample Return Mission}

Christopher W. Brunner

The George Washington University

Joint Institute for Advancement of Flight Sciences

Langley Research Center, Hampton, Virginia

National Aeronautics and

Space Administration 
Available from:

NASA Center for AeroSpace Information (CASI)

7121 Standard Drive

Hanover, MD 21076-1320

(301) 621-0390
National Technical Information Service (NTIS) 5285 Port Royal Road Springfield, VA 22161-2171

(703) 605-6000 


\begin{abstract}
In 2003, NASA solicited proposals for a robotic exploration of the lunar surface. Submissions were requested for a lunar sample return mission from the South Pole-Aitken Basin. The basin is of interest because it is thought to contain some of the oldest accessible rocks on the lunar surface. A mission is under study that will land a spacecraft in the basin, collect a sample of rock fragments, and return the sample to Earth. Because the Aitken Basin is on the far side of the Moon, the lander will require a communications relay satellite (CRS) to maintain contact with the Earth during its surface operation. Design of the CRS's orbit is therefore critical. This paper describes a mission design which includes potential transfer and mission orbits, required changes in velocity, orbital parameters, and mission dates. Several different low lunar polar orbits are examined to compare their availability to the lander versus the distance over which they must communicate. In addition, polar orbits are compared to a halo orbit about the Earth-Moon $\mathrm{L}_{2}$ point, which would permit continuous communication at a cost of increased fuel requirements and longer transmission distances. This thesis also examines some general parameters of the spacecraft systems for the mission under study. Mission requirements for the lander dictate the eventual choice of mission orbit. This mission could be the first step in a period of renewed lunar exploration and eventual human landings.
\end{abstract}




\section{Acknowledgements}

The author would like to thank Robert Dillman of NASA Langley Research Center for his guidance and assistance as well as for answers to numerous questions. Thanks to Professor Robert Blanchard of The George Washington University for his positive support, advising, and allowing the author to take this project where it interested him the most. Thanks also to Dr. Robert Tolson, formerly of The George Washington University, for his insightful ideas. Appreciation to past and current members of the Exploration Engineering Branch at NASA Langley Research Center including Brooke Anderson, Jim Corliss, Doris Hamill, R. Keith Johnson, Herbert Kowitz, Christopher Kuhl, Robert Moses, and Henry Wright for their advice and contributions. Thanks to my family and friends for their continued support. Special thanks go to Erin Myers for her advice and unwavering encouragement. 


\section{Table of Contents}

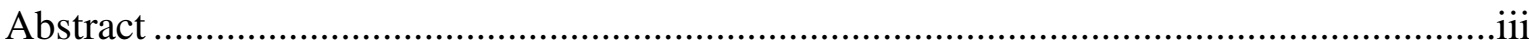

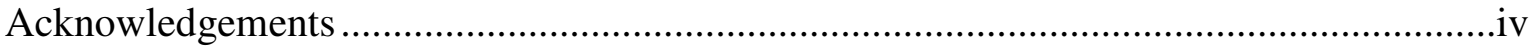

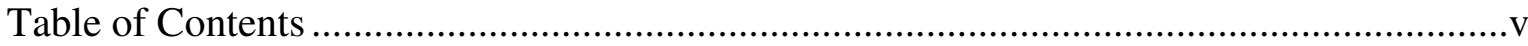

List of Figures ..................................................................................................... vii

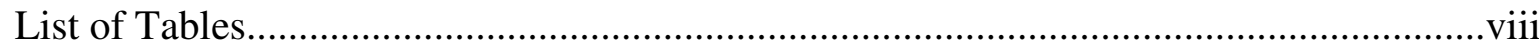

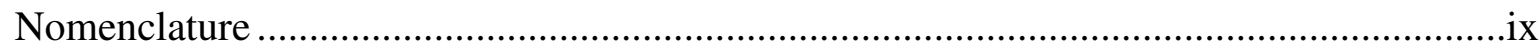

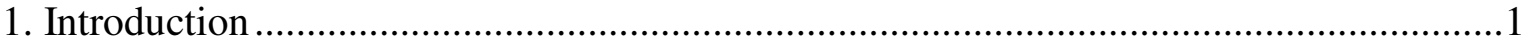

1.1 Motivation and Lunar Sample Return Mission Overview .......................................1

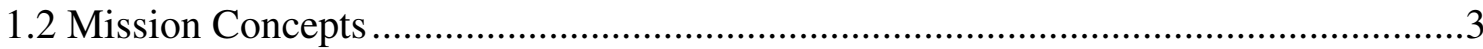

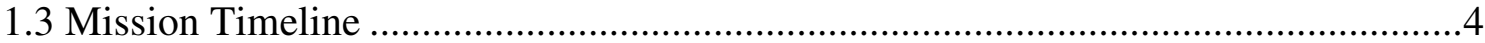

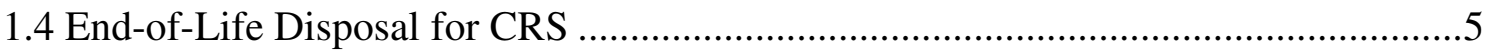

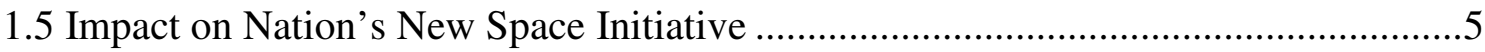

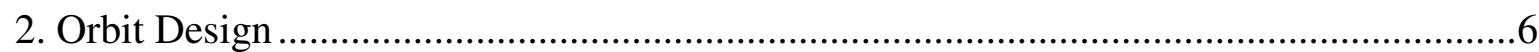

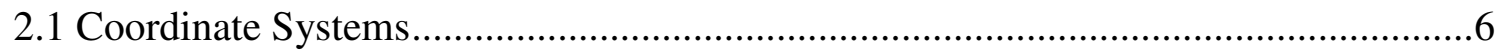

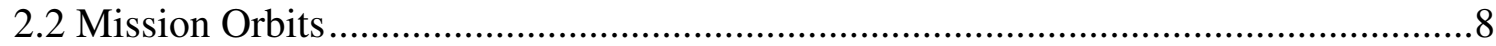

2.2.1 Low-Lunar Polar Orbit Mission Option........................................................ 8

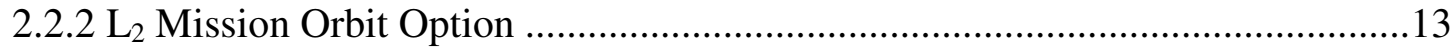

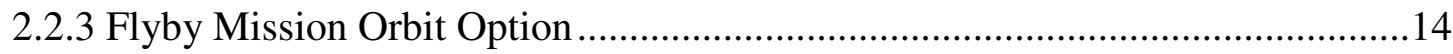

2.3 Transfer Orbit Refinement and Options .......................................................... 14

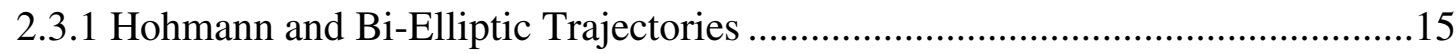

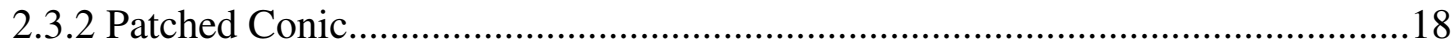

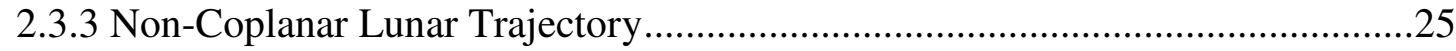

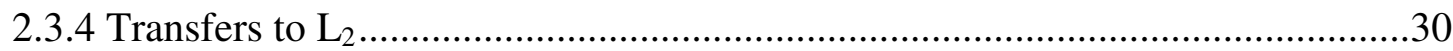




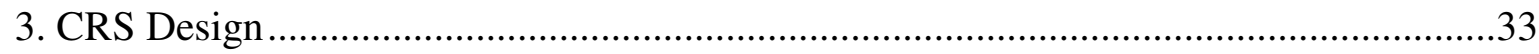

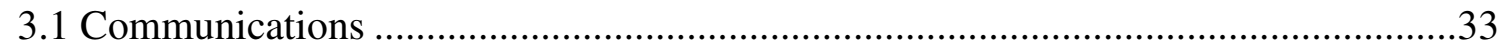

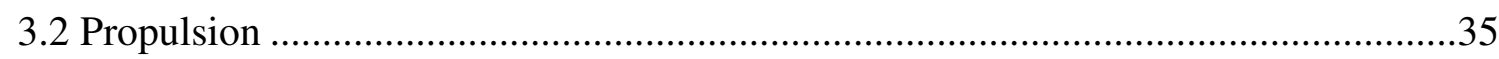

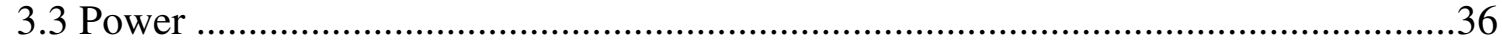

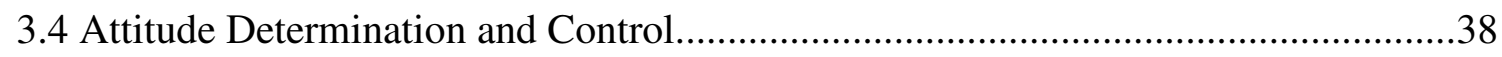

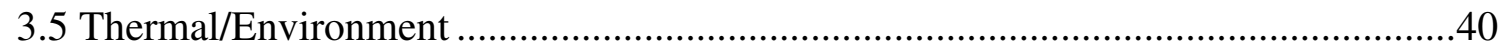

3.6 Command \& Data Handling .................................................................... 41

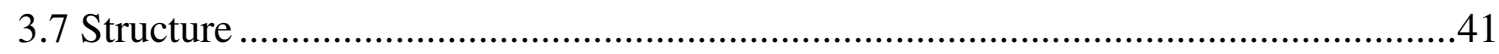

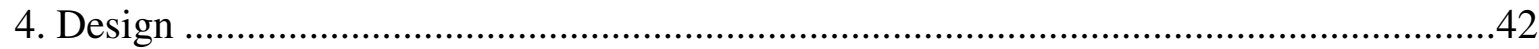

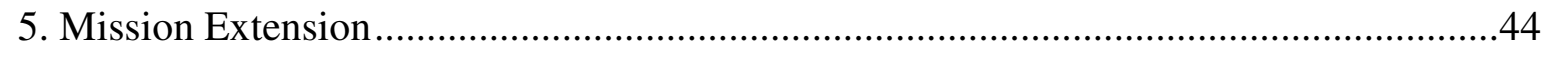

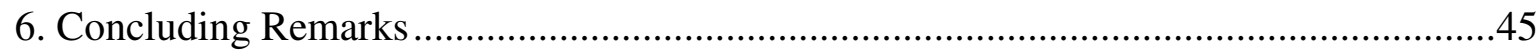

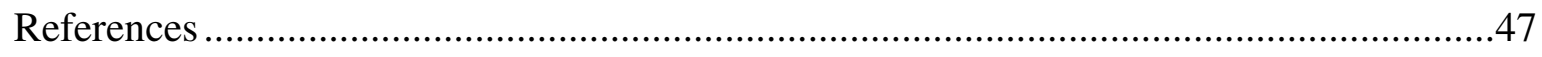

Appendix A-Text of the New Frontiers Announcement of Opportunity Describing the Lunar Mission Science Requirements.............................................................49

Appendix B-Computer Codes (Matlab) .............................................................50 


\section{List of Figures}

Figure 1 Clementine Topographic Map of the Moon .....................................................2

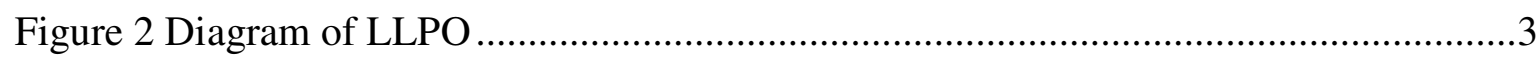

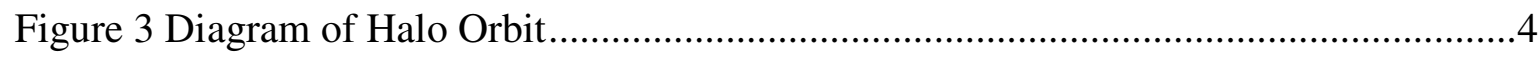

Figure 4 Lunar Coordinate Systems.................................................................

Figure 5 Classical Orbital Elements..........................................................................

Figure 6 Baseline Orbit with Varying Eccentricity .....................................................10

Figure 7 Changing Inclination Versus Fuel Mass ......................................................12

Figure 8 Baseline Orbit with Varying Radius of Perilune ..............................................13

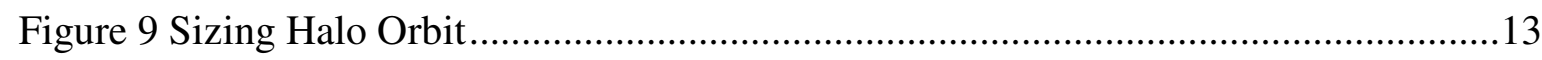

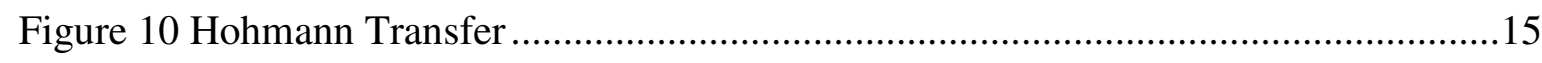

Figure 11 Bi-elliptic Transfer............................................................................. 17

Figure 12 Comparing Hohmann and Bi-Elliptic $\Delta \mathrm{v}$ Requirements and TOF...................17

Figure 13 Patched Conic Transfer ......................................................................... 18

Figure 14 Conditions at the Patch Point.....................................................................2

Figure 15 Summary of $\Delta \mathrm{v}$ and TOF Required for Transfer to LLPO.............................24

Figure 16 Angular Relationships of the Non-Coplanar Lunar Trajectory .........................26

Figure $17 \Delta \mathrm{v}$ and TOF Requirements for Hohmann or Bi-Elliptic $\mathrm{L}_{2}$ Transfer ................31

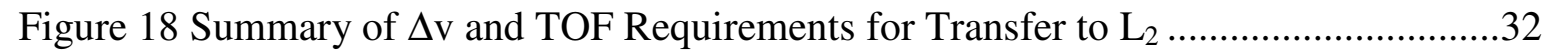

Figure 19 Data Return and Viewing Time for Mission Orbits ......................................35

Figure 20 Fuel Mass as a Function of $\Delta \mathrm{v}$ and Spacecraft Dry Mass ..............................36

Figure 21 Communications Geometry for $\mathrm{L}_{2}$ Halo Orbit .............................................39 


\section{List of Tables}

Table 1 Mission Timelines....................................................................................4

Table 2 Implications of LLPO Eccentricity .......................................................... 11

Table 3 Baseline Mission Orbit.............................................................................. 12

Table 4 Minimum Energy Earth Departure for CRS to Reach Moon SOI ........................23

Table 5 Summary of Patched Conic Conditions .........................................................24

Table 6 Non-Coplanar Lunar Trajectory Conditions Launch Opportunities ......................30

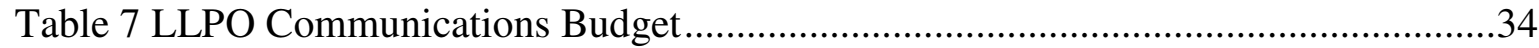

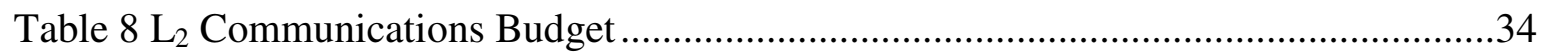

Table 9 Higher Data Rates for Lander to CRS $\mathrm{L}_{2}$ Communications.................................34 


\section{Nomenclature}

$a$

ADCS

AO

C\&DH

CRS

D

DSN

E

e

HGA

$i$

LEO

LLPO

LOI

$\mathrm{L}_{2}$

$\mathrm{p}$

$r_{b}$

$\mathrm{r}_{\mathrm{p}}$

$\mathrm{R}_{\mathrm{s}}$

$\mathrm{r}_{0}$

$\mathrm{r}_{1}$

SOI

STK

$\mathrm{t}_{0}$

$t_{1}$

$t_{c}$

$t_{\text {ff }}$

TOF
Semi-major axis

Attitude determination and control system

Announcement of Opportunity

Command and data handling

Communications relay satellite

Earth-Moon average distance

Deep Space Network

Eccentric anomaly

Eccentricity

High gain antenna

Inclination

Low Earth orbit

Low lunar polar orbit

Lunar orbit insertion

Collinear Earth-Moon Lagrange point on far side of Moon

Semi-parameter

Intermediate radius (for bi-elliptic orbits)

Radius of periapsis

Radius of the sphere of influence

Initial orbit radius of satellite around Earth

Earth-Moon distance at intercept

Sphere of influence

Satellite Tool Kit

Spacecraft launch date and time

Spacecraft and Moon intercept date and time

Coasting time

Free-flight time

Time of flight 


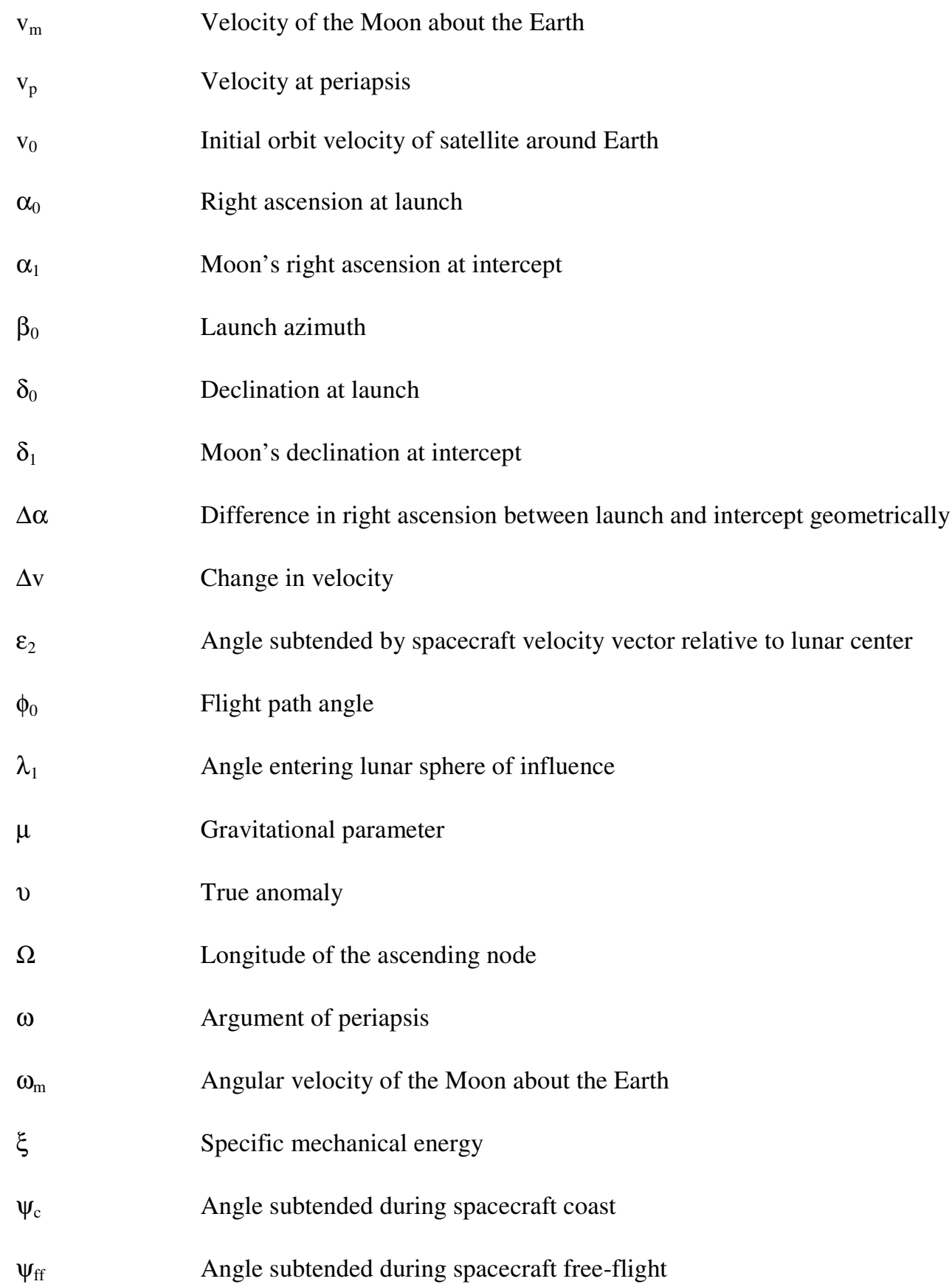




\section{Introduction}

The Moon, our nearest neighbor in the solar system, has drawn the attention of many scientists and engineers in the past. Since December 14, 1972 when the last astronauts left Mare Serenitatis, there has been an interest in returning to the Moon [1]. A limited return has occurred in the form of unmanned space probes. Clementine, Lunar Prospector, Muses A (Hiten), and Smart 1 are completed or ongoing missions [2-5]. In addition, the former Soviet Union launched several missions in the late 1970s [6]. Other future lunar missions are on the drawing board at the current time.

\subsection{Motivation and Lunar Sample Return Mission Overview}

Under the New Frontiers program, NASA is considering a mission to return a sample of lunar rock from the Lunar South Pole-Aitken Basin as one of four possible goals. The purpose of this mission would be to obtain rock fragments from an area on the Moon where scientists believe that some of the oldest rocks on the Moon are readily accessible at the surface [7]. These will be examined for information on the geology and evolution of the early solar system [8]. In addition, NASA would like to return a sample from Mars and this lunar mission could serve as the test-bed for technology and procedures to be used for the eventual Mars sample return mission.

The Lunar South Pole-Aitken Basin challenges the mission design because the landing site is located on the far side of the Moon and does not have line-of-sight communication with the Earth, due to the tidally locked rotation of the Moon about the Earth. Figure 1 shows the position of the Aitken Basin on the Moon [9]. 

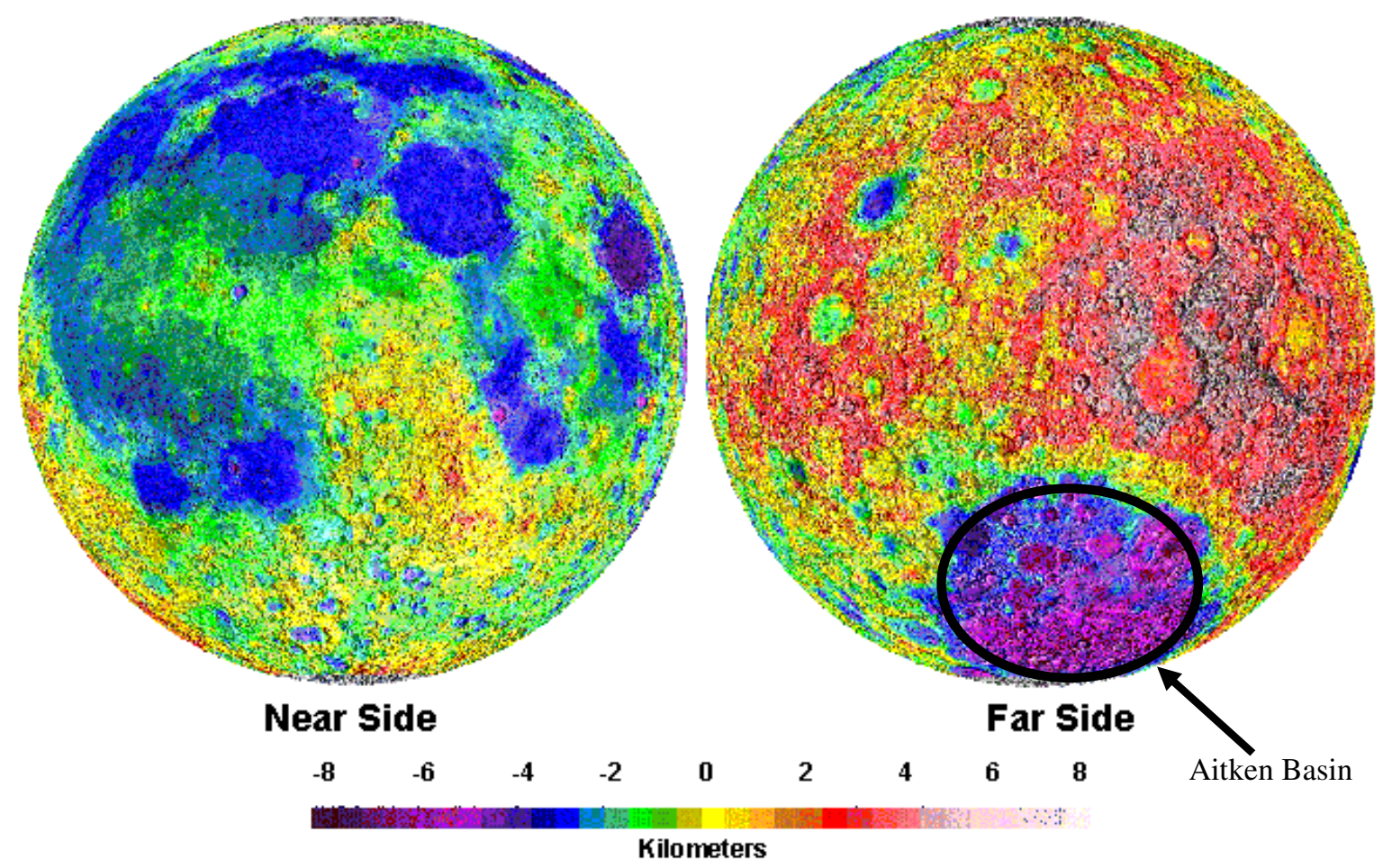

Figure 1 Clementine Topographic Map of the Moon

As a result of the loss of previous missions during communications blackouts, such as Mars Climate Orbiter and Mars Polar Lander, NASA stipulated that "Telemetry coverage of critical events is necessary for analysis and ability to incorporate information in follow-on projects" [10-11]. In other words, for all future missions, spacecraft must have the capability of communicating data about critical mission events back to Earth. This communication does not need to be in real time or in direct contact with the spacecraft. Thus, a lander must have some means of relaying data about the mission events back to Earth at some point. The mission to be examined requires a communications relay satellite (CRS) in an orbit that allows the relay of data between the Earth and the lander, though not necessarily concurrently.

The CRS must be in a position to receive data from the lander and transmit commands during such critical events as approach engine burns to the Moon, landing in the Aitken Basin, important surface activities, and takeoff. NASA may refine which events it considers critical at mission execution. However, the stated events are expected to be included as they are critical to mission success. The CRS is also available to relay routine data from the lander back to Earth or alternatively, the lander could store this data on the sample return capsule for eventual retrieval after its return to Earth and to relay commands from Earth to the lander. 


\subsection{Mission Concepts}

Two CRS scenarios meet the mission requirements. First, the CRS could be placed in a highly inclined low-lunar polar orbit (LLPO). This minimizes the distance between the CRS and the lander during communications periods and simplifies the design of the transfer orbit. However, critical mission events are constrained to times when the CRS is at the point in its orbit whereby it can receive data from the lander. Figure 2 shows a representative LLPO.

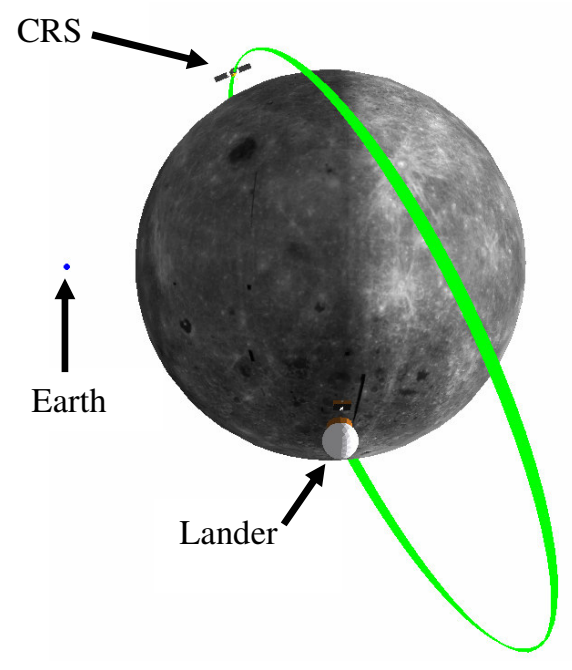

Figure 2 Diagram of LLPO

Alternatively, the CRS could be placed in a halo orbit at the second Earth-Moon Lagrange point $\left(\mathrm{L}_{2}\right)$. This point is an unstable saddle point in the three-body problem for the Earth-Moon-satellite system [12]. This orbit allows the CRS to have $100 \%$ viewing time of both the lander and the Earth. However, it is much further from the surface of the Moon than the LLPO option, $64,500 \mathrm{~km}$ instead of about $300 \mathrm{~km}$, and $16.8 \%$ further from the Earth, $448,900 \mathrm{~km}$ instead of $384,400 \mathrm{~km}$. $\mathrm{L}_{2}$ is always $16.8 \%$ further than the Moon-Earth distance due to the dynamics of the 3-body problem and the mass ratio of the Moon to the Earth. The halo orbit is necessary because the Moon resides directly in the communications path the CRS would use if it were positioned exactly at $\mathrm{L}_{2}$. Further discussions of halo orbits are available in the literature [13]. Figure 3 shows the halo orbit relative to the Earth and Moon and the view of the halo orbit, as it would be seen from Earth. The halo orbit is nearly circular. 


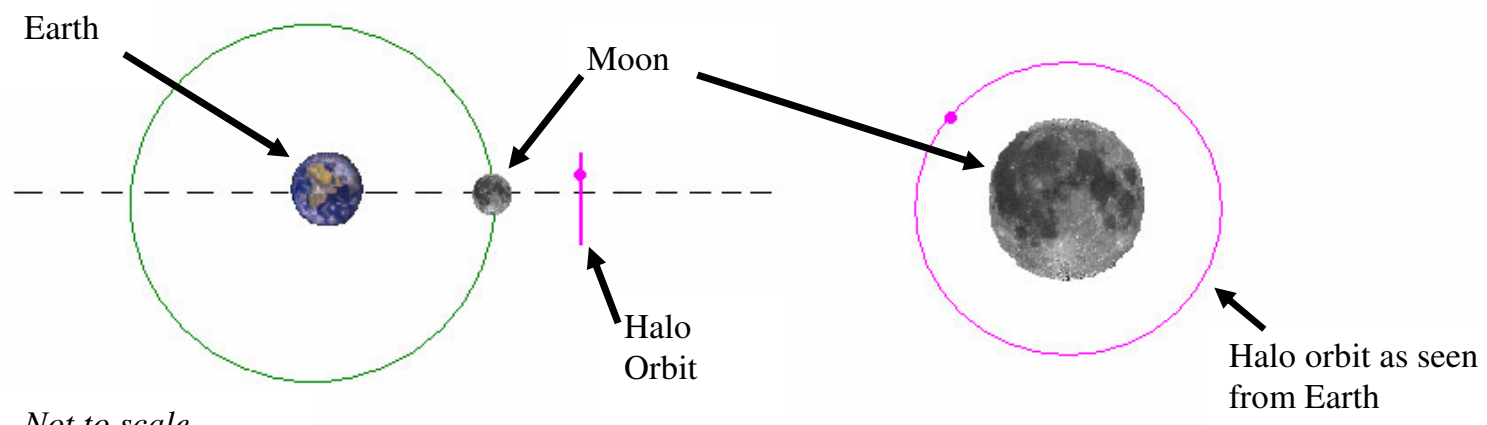

Not to scale

Figure 3 Diagram of Halo Orbit

The eventual choice of mission concept affects designs for the spacecraft systems. The trade-offs will be discussed below. However, issues such as cost and scheduling will not be addressed directly in this study.

\subsection{Mission Timeline}

There are several potential timelines for the mission that meet the requirements of a NASA Announcement of Opportunity (AO) [8]. See Appendix A for discussion of the lunar mission requirements. All of them take place around the time of new Moon so that the lander may gather solar power while it is on the surface. Staying for the lunar night is not an option, as it requires prohibitively large batteries to run the required survival heaters. Two potential timelines are outlined in Table 1.

Table 1 Mission Timelines

\begin{tabular}{|l||c|c|}
\cline { 2 - 3 } \multicolumn{1}{c|}{} & Lunar Apogee & Lunar Perigee \\
\hline Launch: & December 5, 2009 & June 1, 2010 \\
\hline Moon arrival: & December 9, 2009 & June 5, 2010 \\
\hline New Moon: & December 16, 2009 & June 12, 2010 \\
\hline Operational Dates: & December 9-24, 2009 & June 5-20, 2010 \\
\hline Sample Return by: & December 30, 2009 & June 26, 2010 \\
\hline
\end{tabular}

The first timeline begins with a launch on December 5, 2009, the operation of the CRS from the $9^{\text {th }}$ to the $24^{\text {th }}$, and a return to Earth by the $30^{\text {th }}$. The second begins with a launch on June 1,2010 , the operation of the CRS from the $5^{\text {th }}$ to the $20^{\text {th }}$, and a return by the $26^{\text {th }}$. There are some advantages and disadvantages to choosing between these two timelines. First, slightly greater $\Delta v$, about $1 \%$, is required to reach the Moon at perigee. However, the Moon is about $14 \%$ closer, $356,000 \mathrm{~km}$ versus $406,200 \mathrm{~km}$, thus requiring less power for the spacecraft to communicate with Earth. These timelines are for the simplest case of a sample return mission whereby Hohmann 
transfers are the primary method of transit. More complicated looping orbits result in earlier launch dates so that the CRS has enough time to reach the Moon before operation.

Other possible dates for perigee encounter include the new Moons of April and May of 2009. Apogee possibilities include the new Moons of November of 2009 and January of 2010. During these landing dates, the Moon-Sun angle may be slightly higher, thus giving the lander a greater amount of solar power, but probably not enough to make a significant difference in solar array size. The sun angle could also be adjusted by landing closer to the Moon's equator or the solar panels could be motorized to track the Sun.

There exist mission dates outside the requirements of the $\mathrm{AO}$ when the Moon is at more extreme perigee and apogee distances, thus extending the communications capabilities or reducing the fuel usage.

\subsection{End-of-Life Disposal for CRS}

The CRS could be configured for long-term usage by having large solar panels, batteries, more fuel for station keeping, and additional efficiency improvements. In any case, before the fuel runs out, the CRS would be directed to crash into the Moon to mitigate any future collision risks. Mission planners may determine that movement to a higher lunar orbit is preferable or from $\mathrm{L}_{2}$ the spacecraft could also enter a solar orbit for end-of-life disposal. Departure from a low-lunar orbit requires significantly more $\Delta \mathrm{v}$ than from an $\mathrm{L}_{2}$ halo orbit since the Lagrange point is unstable.

\subsection{Impact on Nation's New Space Initiative}

With the President's new proclamation for a return of humans to the Moon and Mars, this mission could be a significant first step in re-establishing a foothold on the Moon. The examination of lunar samples may provide information about valuable resources that could be harvested from the Moon. Additionally, the CRS could be made available to provide added communication capabilities for astronauts on the Moon. 


\section{Orbit Design}

There are several goals for the mission which impact CRS orbit design. The first goal is to maximize data transferred from the lander back to Earth. The lander is likely to take hundreds of pictures of its surroundings and of the rock it scoops, which requires many gigabytes of data to be returned [14]. This goal requires balancing viewing time of the lander and distance to the relay satellite. For example, placing the CRS in a highly eccentric LLPO with the apolune of the CRS orbit placed above the landing site, would allow the ground track of the CRS to dwell over the lander for a long time to transmit data, but the transfer would occur at slower rates due to power and signal strength losses at the greater distance. In a less eccentric orbit, the CRS is not in view of the lander as long, but it can be significantly closer thus allowing for greater data transfer rates. The viewing time and transfer rate issue is not of great concern for Earth communications since the capabilities of Earth's facilities are much greater. The second goal is to minimize the required $\Delta \mathrm{v}$ and thus the overall fuel necessary for injection into the transfer orbit. Additional fuel translates into more mass, which requires a larger launch vehicle to place the CRS into orbit, which in turn costs more-though cost is not an issue to be addressed directly in this paper. The third goal is to minimize the time of flight (TOF) for the transfer orbit. This minimizes operations cost because engineers must monitor the spacecraft during the period of transfer. Furthermore, a longer TOF increases the probabilities of a component malfunction.

\subsection{Coordinate Systems}

The landing site is assumed to be at $55^{\circ} \mathrm{S}$ and $180^{\circ} \mathrm{E}$. This location is approximately in the middle of the Aitken Basin, which ranges from $20^{\circ} \mathrm{S}$ to $90^{\circ} \mathrm{S}$ and $140^{\circ} \mathrm{E}$ to $150^{\circ} \mathrm{W}$. Coordinates on the Moon are similar to those on Earth, that is, the lunar equator is in a plane perpendicular to the axis of rotation. North is in a similar celestial direction as Earth, pointed somewhat in the direction of celestial north, but variable depending on several orbital parameters. Zero degrees longitude is centered in the middle of the visible Moon, in the direction of Earth (averaged for the Moon's slight rotation from the perspective of Earth). East longitude is towards sunrise if you are standing on the Moon (to the right of center if you are standing on the Earth looking at the Moon with north towards the top) [15].

The coordinate system used for analysis of the LLPO mission orbit is the "Moon Fixed Axes." In this coordinate system, $\mathrm{Z}$ points along the mean rotation axis of the Moon, $\mathrm{X}$ points toward $0^{\circ}$ longitude (generally 
towards Earth) and $\mathrm{Y}$ points to $90^{\circ} \mathrm{E}$, thus completing the right-handed coordinate system [15]. The ascending node of the orbit is measured from the positive $\mathrm{X}$-axis. Figure 4 shows the lunar coordinate systems. Figure 5 shows the classical orbital elements [12].
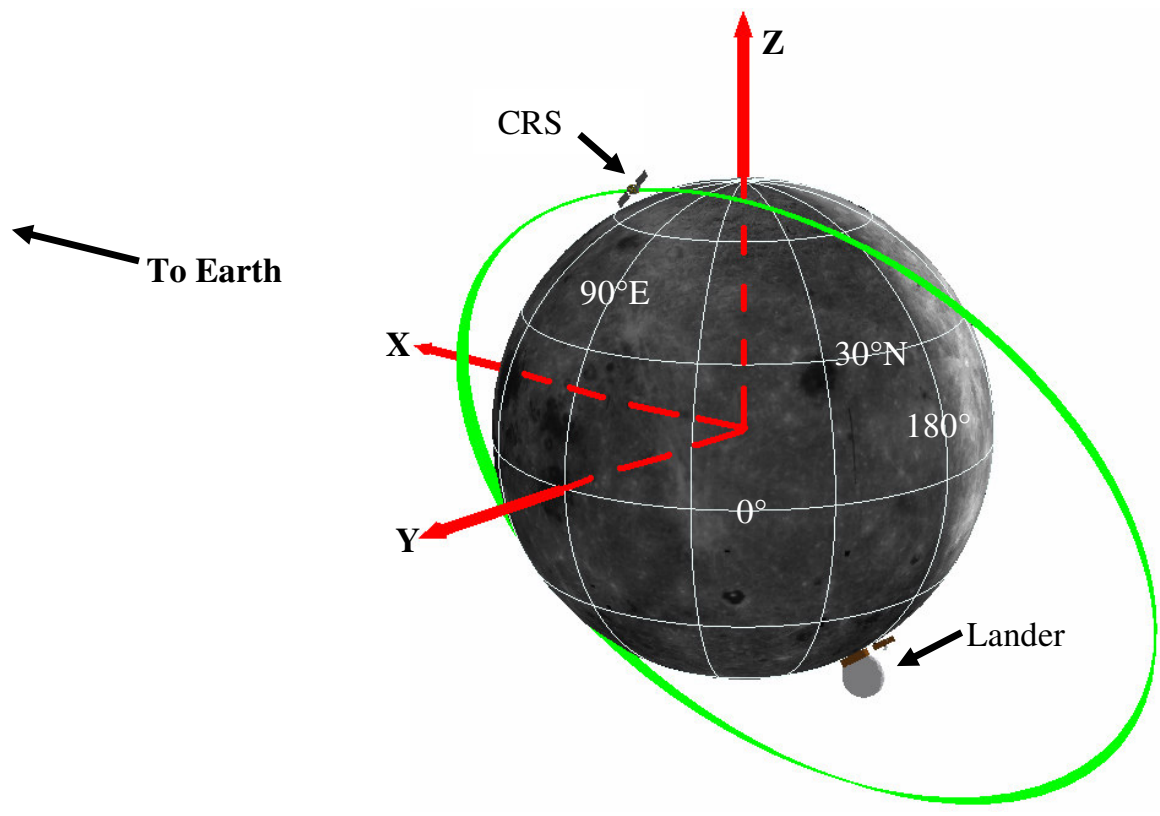

Figure 4 Lunar Coordinate Systems

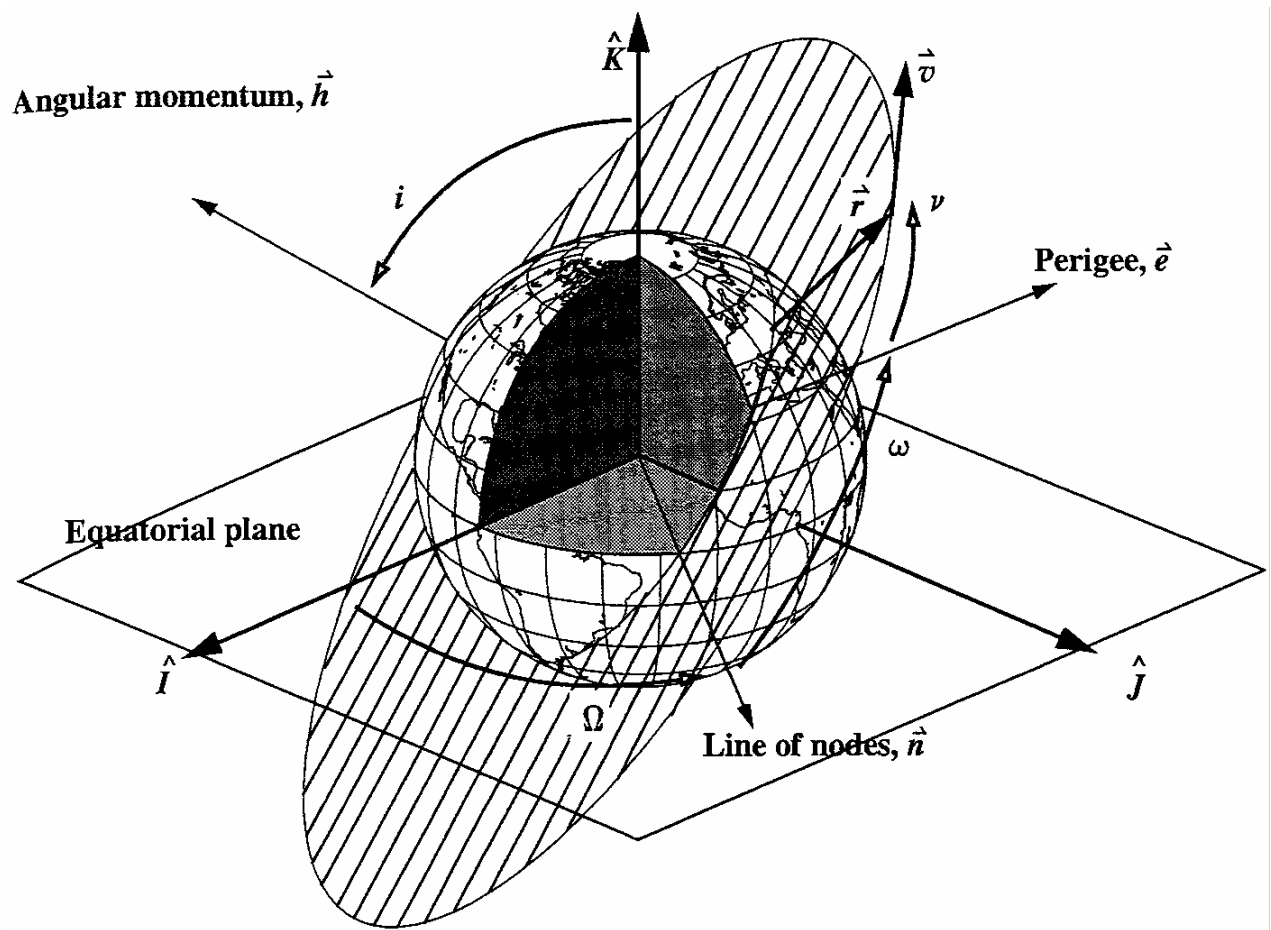

Figure 5 Classical Orbital Elements 


\subsection{Mission Orbits}

Several different mission orbit options including low-lunar polar orbit (LLPO), $\mathrm{L}_{2}$ halo orbit, and flyby are examined herein. Other possibilities are to station three or four satellites around the Moon in a network, which would be very expensive, or to use an existing satellite in geosynchronous Earth orbit to communicate with the lander, which would constrain the landing site to a location near the Moon's South Pole. Examination of all the possibilities for lander communications is outside the scope of this research.

\subsubsection{Low-Lunar Polar Orbit Mission Option}

One of the options for the CRS is to enter a low-lunar polar orbit. The precise details of entering a given orbit from the transfer orbit are outside the scope of this paper and are left to a full-scale mission design with all the accompanying constraints. Several example orbits are presented along with a variety of orbit parameters that can be varied to get an orbit with the desired characteristics.

This initial design is created using Satellite Tool Kit (STK) [15]. The orbit epoch is set for December 9, 2009 at 06:00:00 UTC, as this is the presumed date of lunar orbit insertion. The spacecraft is assumed to be in sight of the lander when it is $5^{\circ}$ above the horizon. For Earth satellites, this requirement is typically due to viewing obstacles such as trees, buildings, and mountains. However, on the Moon, terrain should be the only obstacle to communication. In STK, this is accomplished using a "sensor" on the lander with an $80^{\circ}$ cone field of view. The remaining $5^{\circ}$ is at both horizons. The CRS is free to communicate in any direction since it can reorient its antenna and due to the absence of nearby obstructions.

Orbit size and shape were the essential parameters to vary in this design, thus they are defined last. Inclination, an orbit orientation parameter, is examined first. The inclination is measured from the Z-axis to the angular momentum vector of the orbit. Since the lander is assumed to be at the center of the Aitken Basin at $55^{\circ} \mathrm{S}$ latitude on the Moon, a fairly high orbital inclination is necessary to enable the CRS to view the lander. An iterative design with STK shows that an inclination of $55^{\circ}$ is the minimum inclination necessary and allows the CRS to cover the full width of the sky based above the lander, but these passes shift slightly as the orbit precesses due to the rotation of the Moon. A higher inclination of about $65^{\circ}$ is desirable due to the negative precession. Next, the other orbit orientation parameter, longitude of ascending node $(\Omega)$ is examined. The node is measured in the right-handed 
positive direction about the $\mathrm{Z}$-axis with the $\mathrm{X}$-axis being zero degrees. The initial positioning of the node is constrained so that the CRS remains visible by the lander during the mission. As the Moon slowly rotates, the node of the orbit precesses about the Moon's Z-axis in a negative direction by $1.53 \times 10^{-4} \mathrm{deg} / \mathrm{s}$. STK calculations show that a node initially placed $11^{\circ}$ from the positive $\mathrm{X}$-axis met these constraints. Next, the argument of periapsis ( $\omega)$ for the non-circular orbit orients the orbit such that the perilune is directly nadir to the lander, above $55^{\circ} \mathrm{N} 0^{\circ} \mathrm{E}$, at the midpoint of the mission period. Since the mission is assumed to last from December 9-24, 2009, the perilune is nadir to the lander on December 16, 2009. This places the apolune of the orbit directly zenith to the lander at that time, in order to maximize communications time. This is due to Kepler's second law: as the spacecraft traverses the portion of its orbit that is further from the central body, its velocity is slower. The final angular measure of spacecraft location within an orbit, the true anomaly $(v)$ is not of great consequence to this analysis and is set to zero degrees at the orbit epoch.

Orbit shape and size are interrelated in this design. First, the radius of perilune $\left(r_{p}\right)$ is fixed at $2000 \mathrm{~km}$. Again, this was nadir to the lander. The critical design parameter is the orbit eccentricity $(e)$. The choice of $r_{p}$ and $e$ dictates the semi-major axis ( $a$ ) by equation (1).

$$
a=r_{p} /(1-e)
$$

Fig. 6 shows how eccentricity changes the viewing times, given in percentages of total epoch time and change in average distance from the lander to the CRS while it is viewable. To give an idea of what the percentages of viewing time correspond to, $100 \%$ viewing is 356 hours and $50 \%$ is 178 hours. These encompass the previously defined mission operation dates in Table 1. 


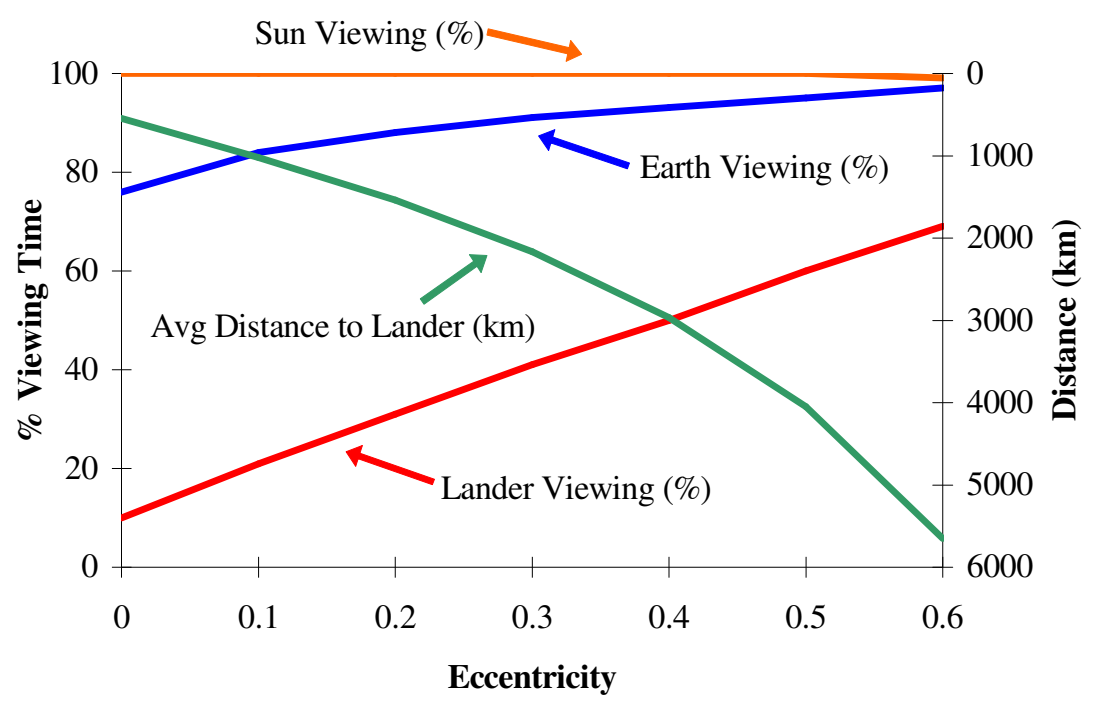

Figure 6 Baseline Orbit with Varying Eccentricity

The major variable in this design is orbit eccentricity. With apolune positioned directly above the lander, lower eccentricities produce closer passes of the lander, permitting a higher data rate for the same communication equipment, but lower eccentricities also give the CRS less viewing time of the lander per pass. Due to the high inclination and positioning of the orbit, the Sun is eclipsed rarely. Only when the inclination, radius of perilune, and eccentricity are not at their extremes, do eclipses occur. Some battery backups are necessary at high eccentricities. However, sunlight conditions are expected for a majority of possible orbits. Some of the viewing time for Earth and the lander occurs simultaneously. Although this is not quantified in this study, some eclipses of both the Earth and the lander do occur. Also, the CRS approaches the Moon on a hyperbolic trajectory $(e>1)$, so more fuel will be required to reach a low eccentricity orbit than a high one. Table 2 summarizes the eccentricity trade-offs and implications. 
Table 2 Implications of LLPO Eccentricity

\begin{tabular}{|l|l|l|l|}
\cline { 2 - 4 } \multicolumn{1}{l|}{ Viewing time } & Low $e(0-0.2)$ & High $e(0.3-0.6)$ & Implications \\
\hline Distance & Less per pass and overall & $\begin{array}{l}\text { Greater per pass and } \\
\text { overall }\end{array}$ & $\begin{array}{l}\text { Time for engineers on } \\
\text { ground to make } \\
\text { decisions, total time for } \\
\text { data transmission }\end{array}$ \\
\hline Sun eclipses & Shorter & Longer & $\begin{array}{l}\text { Power req. for } \\
\text { transmitter }\end{array}$ \\
\hline Earth eclipses & Few at low inclinations & Few at low inclinations & $\begin{array}{l}\text { Size of batteries and } \\
\text { thermal design and } \\
\text { control methods }\end{array}$ \\
\hline Entry trajectory & $\begin{array}{l}\text { Significant change } \\
\text { to course necessary }\end{array}$ & $\begin{array}{l}\text { Maintains high } e \text { of } \\
\text { hyperbolic entry }\end{array}$ & $\begin{array}{l}\text { Length of continuous } \\
\text { comm. from lander to } \\
\text { Earth }\end{array}$ \\
\hline
\end{tabular}

The other orbital parameters can be varied in a manner similar to eccentricity. For instance, it takes a significant amount of fuel to enter an orbit around the Moon with a high inclination, but a lower inclination has less viewing time. This result concerning greater fuel requirement for higher inclination is obtained by applying equations (2) and (3)

$$
\begin{gathered}
\Delta v_{i}=2 v_{\text {initial }} \cos \left(\phi_{f p a}\right) \sin \left(\frac{\Delta i}{2}\right) \\
m_{p}=m_{f}\left(e^{\left(\Delta v / I_{s p} g\right)}-1\right)
\end{gathered}
$$

In equation (2), $\Delta v_{i}$ is the change in velocity required for a particular inclination change only, $v_{\text {initial }}$ is the initial velocity of the spacecraft, which is $1.57 \mathrm{~km} / \mathrm{s}$ for the baseline mission orbit with an orbital radius of $2000 \mathrm{~km}$, $\phi_{f p a}$ is the flight path angle, assumed to be zero degrees, and $\Delta i$ is the change in inclination [12]. In equation (3), $g$ is the gravity at the Earth's surface $\left(9.81 \mathrm{~m} / \mathrm{s}^{2}\right), I_{s p}$ is the specific impulse of the rocket fuel (assumed to be a liquid propulsion system with $I_{s p}$ of $300 \mathrm{~s}$ ), $m_{f}$ is the final spacecraft mass (also referred to as the dry mass) assumed to be 100 kilograms, and $m_{p}$ is the mass of the propellant necessary to make a certain $\Delta \mathrm{v}$ [16]. Figure 7 shows the fuel requirement for inclination changes. 


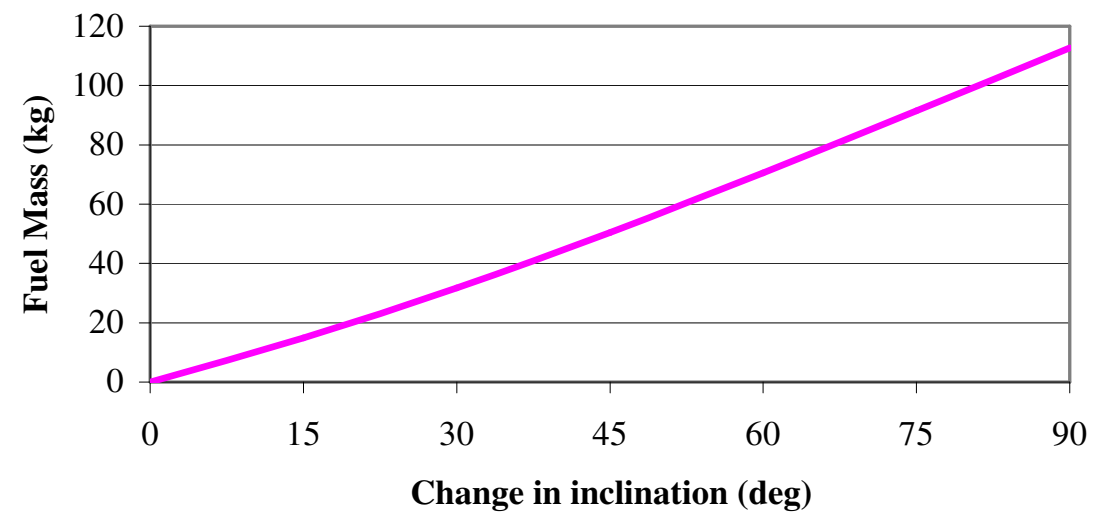

Figure 7 Changing Inclination versus Fuel Mass

A summary of the baseline mission orbit for the epoch Dec 9, 2009 9:00:00 UTC to Dec 24, 2009 5:00:00 UTC, a period in which the landing location is illuminated by sunlight, appears in Table 3 .

Table 3 Baseline Mission Orbit

\begin{tabular}{|c|c|c|c|c|c|}
\hline $\mathrm{r}_{\mathrm{p}}$ & $\mathrm{e}$ & $\mathrm{i}$ & $\Omega$ & $\omega$ & $\mathrm{v}$ \\
\hline $2000 \mathrm{~km}$ & 0 & $65^{\circ}$ & $11^{\circ}$ & $80^{\circ}$ & $0^{\circ}$ \\
\hline
\end{tabular}

If the baseline mission orbit is changed, the average distance from the lander during viewing and the total viewing time changes. The decision about which orbit to choose is best left to a mission planner, but some suggestions are included in further discussion.

For a given eccentricity, some slight variation of inclination maximizes the viewing time. For this study, all of the data are for a fixed inclination of $65^{\circ}$. For fixed communications components, as the average distance increases, the data rate must decrease in order to maintain a communications link. Since viewing time increases, it might appear that the same total data can be returned; however, this is not the case, since the relationship is not linear. Despite a decrease in viewing time percentage by going to a lower eccentricity, the data rate can increase by a factor of two with a decrease in average viewing distance by several hundred kilometers. The total data returned is much greater from shorter distances. Further discussion of data return appears in Section 3.1.

The radius of perilune, for a fixed eccentricity of 0.3 , also slightly affects the viewing time and average distance. The extreme variations encountered in viewing time when eccentricity is varied are not as evident when the radius of perilune is varied. However, the average distance changes by a similar amount, as shown in Figure 8 . 


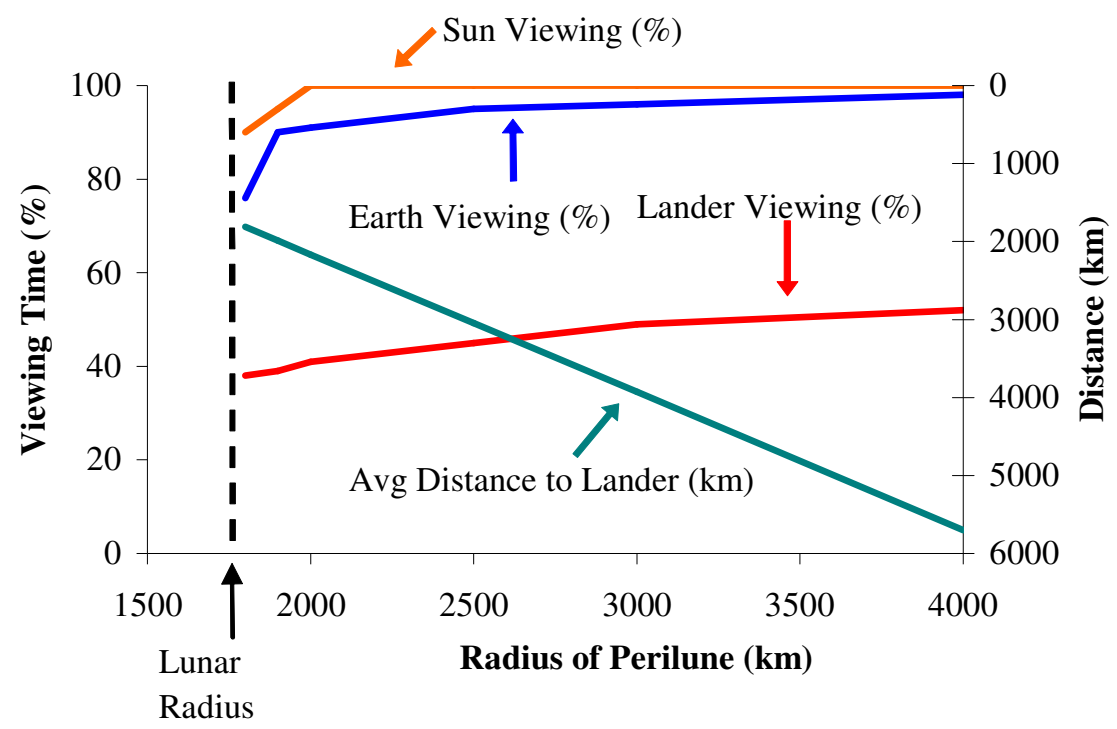

\section{Figure 8 Baseline Orbit with Varying Radius of Perilune}

\subsubsection{Mission Orbit Option}

For this option, the CRS is placed in a halo orbit about the Earth-Moon Lagrange point $\left(\mathrm{L}_{2}\right)$. This point is on the far side of the Moon. The orbit resides in a plane that is perpendicular to a line drawn between the Earth and the Moon (see Fig. 3). An orbital radius of slightly less than twice the lunar radius (3100 km) is required so that the Moon never occults the CRS from Earth. Fig. 9 shows the sizing of the halo orbit.

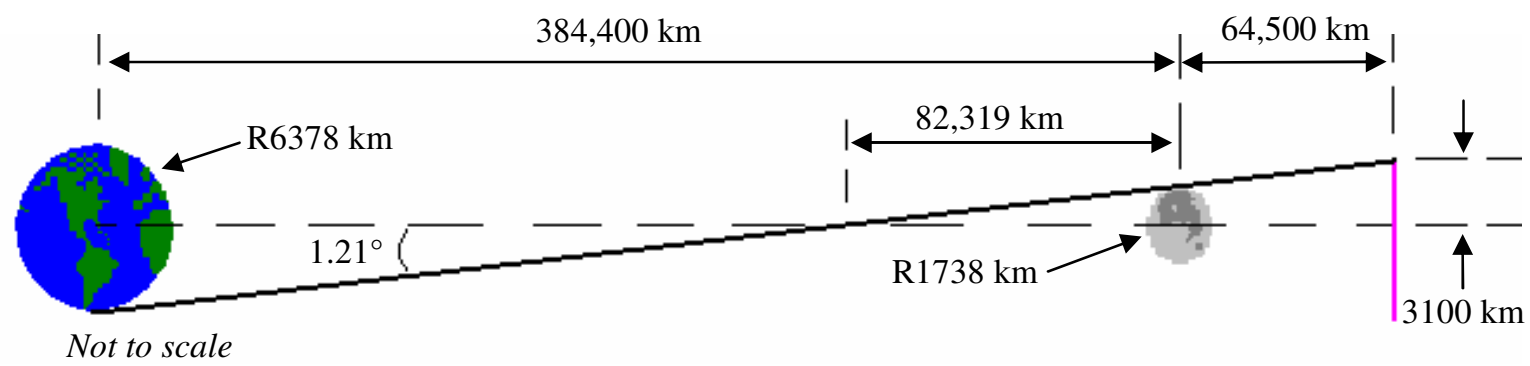

Figure 9 Sizing Halo Orbit

This is when the Moon is at its average distance from the Earth. Brief eclipses of the Sun are possible, but only in the case of an extended mission-none occur during the primary lander-Earth communications relay mission. The average distance from the lander to the Earth-Moon $\mathrm{L}_{2}$ point is $64,500 \mathrm{~km}$, as compared to the distance of 1800 $5700 \mathrm{~km}$ in LLPO. 
For the $\mathrm{L}_{2}$ mission, the CRS has no communications blackouts of the Earth and at worst brief ones of the lander, depending on the landing latitude. Assuming a perfectly spherical Moon and the landing latitude not at the assumed $55^{\circ} \mathrm{S}$, blackouts can occur. To be conservative, the lander should be some distance north of $85^{\circ} \mathrm{S}$ in a site with no local obstructions of the view to the north so that the CRS rises some distance above the horizon to avoid problems caused by local terrain. This constrains the landing latitude for the $\mathrm{L}_{2}$ mission orbit option.

\subsubsection{Flyby Mission Orbit Option}

One final mission orbit option is the flyby. In this case, the CRS simply passes the Moon on a hyperbolic trajectory that allows it to contact the lander and Earth to transfer data. For instance, the CRS and lander could depart Earth orbit under the same power at the same time. Then, the two separate at some point during their transit to the Moon. The lander carries a propulsion system to allow it to enter lunar orbit and subsequently land on the Moon. The CRS simply passes by the Moon. The advantage of this option is that the CRS does not need to carry fuel to enter LLPO. Unfortunately, during the time the lander spends entering orbit and landing, the CRS traverses a great distance away from the Moon thus over-extending the capabilities of its communication system. Viewing of the lander, Earth, and Sun are excellent from this perspective. However, within one day of the operation start, the CRS reaches the same distance as the $\mathrm{L}_{2}$ halo orbit and increases to a distance of over 630,000 $\mathrm{km}$ by the end of operation due to the nature of the hyperbolic trajectory. For this reason, the feasibility of this option requires further investigation as to whether all critical events can be encompassed before transmission becomes impractical.

\subsection{Transfer Orbit Refinement and Options}

Transfer orbits to LLPO and $\mathrm{L}_{2}$ are examined on several levels. For LLPO, the first approximation calculates Hohmann and bi-elliptic trajectories from low-Earth orbit to the distance of the Moon's orbit. Second, the patched conic approximation is applied to the problem of bringing the CRS close to the Moon [17]. Third, a study of non-coplanar lunar trajectories is completed. For transfer to $\mathrm{L}_{2}$, Hohmann and bi-elliptic trajectories are examined.

The AO requires the mission to be launched by June 30, 2010 [8]. Roughly every 29 days a new Moon occurs. The new Moon coincides with perigee or apogee at less frequent intervals. This is not a firm requirement for the mission to take place during the coincidence. Whichever landing date is chosen, the CRS should arrive in position several days before the chosen landing date in order to provide coverage during the landing. 


\subsubsection{Hohmann and Bi-Elliptic Trajectories}

Hohmann transfers are typically the minimum change in velocity between two circular orbits. Depending on the orbital radii, bi-elliptic transfers can require less $\Delta \mathrm{v}$ than the Hohmann case, but take significantly longer [12]. Both are examined.

The Hohmann transfer makes two changes to spacecraft velocity. The first $\Delta \mathrm{v}$ occurs in low-Earth orbit (LEO), at the perigee of the elliptic transfer orbit. The second occurs when the CRS is at the apogee of the transfer orbit, where the distance from Earth coincides with the lunar distance. See Fig. 10 for a representative Hohmann transfer.

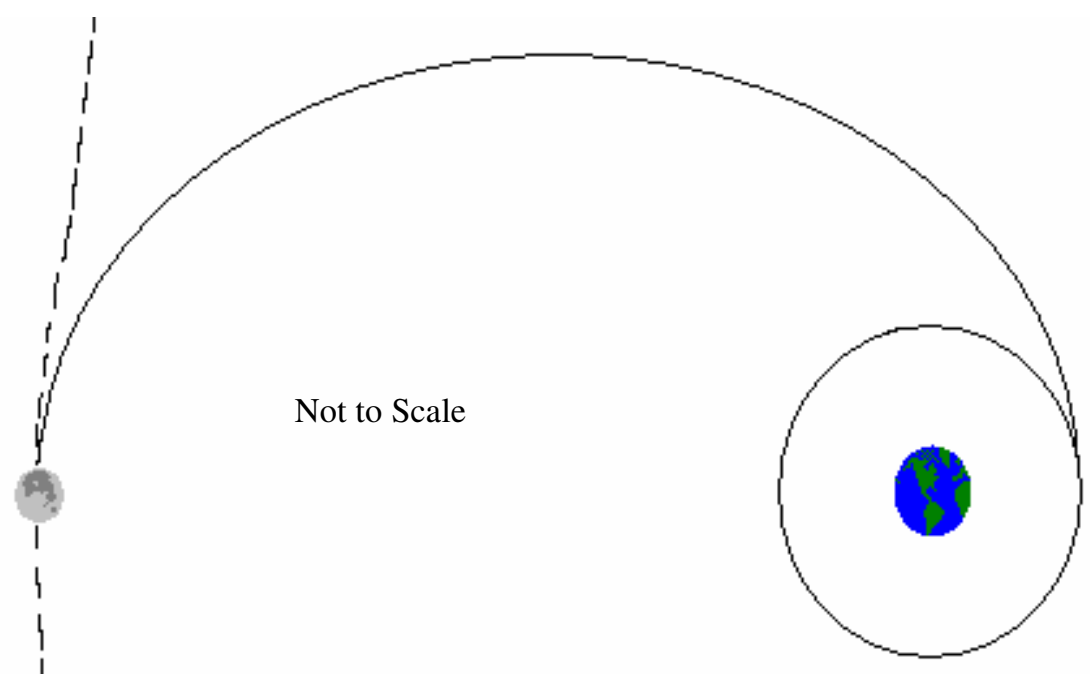

Figure 10 Hohmann Transfer

The Hohmann covers the simplest case of a transfer between two circular orbits. In order for a transfer to take place, the spacecraft begins by orbiting the central body at in an initial radius $\left(r_{\text {initial }}\right)$ with some velocity $\left(v_{\text {initial }}\right)$. The desired final radius $\left(r_{\text {final }}\right)$ has a corresponding final velocity $\left(v_{\text {final }}\right)$. The velocities are found from equations (45). (Where $\mu$ is the gravitational parameter of the central body, Earth).

$$
\begin{aligned}
& v_{\text {initial }}=\sqrt{\frac{\mu}{r_{\text {initial }}}} \\
& v_{\text {final }}=\sqrt{\frac{\mu}{r_{\text {final }}}}
\end{aligned}
$$


(Note: The discussion assumes that $r_{\text {initial }}<r_{\text {final }}$, which implies that $v_{\text {initial }}>v_{\text {final }}$. This is not a necessary condition in general, but it will simplify the discussion here.) The transfer orbit is an elliptical orbit where the perigee coincides with the initial radius and the apogee coincides with the final radius. To determine the size of the transfer orbit $\left(a_{\text {trans }}\right)$, equation (6) is used.

$$
a_{\text {trans }}=\frac{r_{\text {initial }}+r_{\text {final }}}{2}
$$

Once the transfer orbit size has been found, the velocity at perigee $\left(v_{\text {trans }_{a}}\right)$ and the velocity at apogee $\left(v_{\text {trans }_{b}}\right)$ are found from equations (7-8).

$$
\begin{aligned}
& v_{\text {trans }_{a}}=\sqrt{\frac{2 \mu}{r_{\text {initial }}}-\frac{\mu}{a_{\text {trans }}}} \\
& v_{\text {trans }_{b}}=\sqrt{\frac{2 \mu}{r_{\text {final }}}-\frac{\mu}{a_{\text {trans }}}}
\end{aligned}
$$

The necessary $\Delta v$ is found by equation (9).

$$
\Delta v=\left(v_{\text {trans }_{a}}-v_{\text {initial }}\right)+\left(v_{\text {final }}-v_{\text {trans }_{b}}\right)
$$

The time-of-flight (TOF) for the transfer can be found from equation (10).

$$
\tau_{\text {trans }}=\pi \sqrt{\frac{a_{\text {trans }}^{3}}{\mu}}
$$

For the bi-elliptic case, the CRS completes two Hohmann transfers and three changes to spacecraft velocity. It first transfers into an elliptical orbit with apogee at radius $\left(\mathrm{r}_{\mathrm{b}}\right)$, which is greater than the desired final radius $\left(r_{\text {final }}\right)$. Then a second elliptical orbit is used to transfer into the final circular orbit. The first $\Delta \mathrm{v}$ occurs in low-Earth orbit (LEO), at the perigee of the elliptic transfer orbit. The second occurs at the intermediate radius ( $\mathrm{r}_{\mathrm{b}}$ ). The third occurs when the CRS is at the perigee of the second transfer orbit, where the distance from Earth coincides with the lunar distance. The equations above are used again for this calculation. Fig. 11 shows a representative bielliptic transfer. 


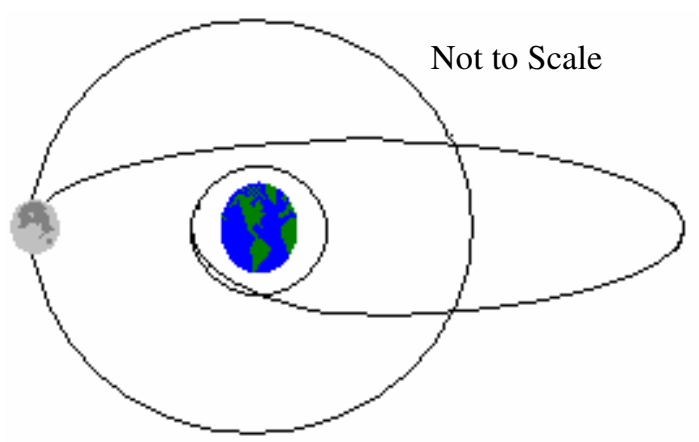

Figure 11 Bi-elliptic Transfer

Calculations for the Hohmann and bi-elliptic transfers are done strictly in the 2-body sense, that is, they assume no other perturbations such as lunar gravity, non-spherical gravity terms of the Earth, atmospheric drag, nonimpulsive maneuvers, solar pressure, and perturbations due to the gravity of Sun, Jupiter, etc. Later in this paper, the patched conic approximation is used to take into account some of the effect of the Moon's gravity and to provide a more realistic approximation.

For this design, it is assumed that the CRS is launched into a $300 \mathrm{~km}$ altitude Earth parking orbit ( $a=6678$ $\mathrm{km})$. The final orbit radius is the average distance from the Earth to the Moon $(384,400 \mathrm{~km})$. The $\Delta \mathrm{v}$ and time of flight (TOF) for the Hohmann transfer and for the bi-elliptic transfer, of which three different intermediate radii are chosen, appear in Figure 12. The intermediate radii are: $\mathrm{r}_{\mathrm{b} 1}=4 \times 10^{5} \mathrm{~km}, \mathrm{r}_{\mathrm{b} 2}=6 \times 10^{5} \mathrm{~km}, \mathrm{r}_{\mathrm{b} 3}=1 \times 10^{6} \mathrm{~km}$.

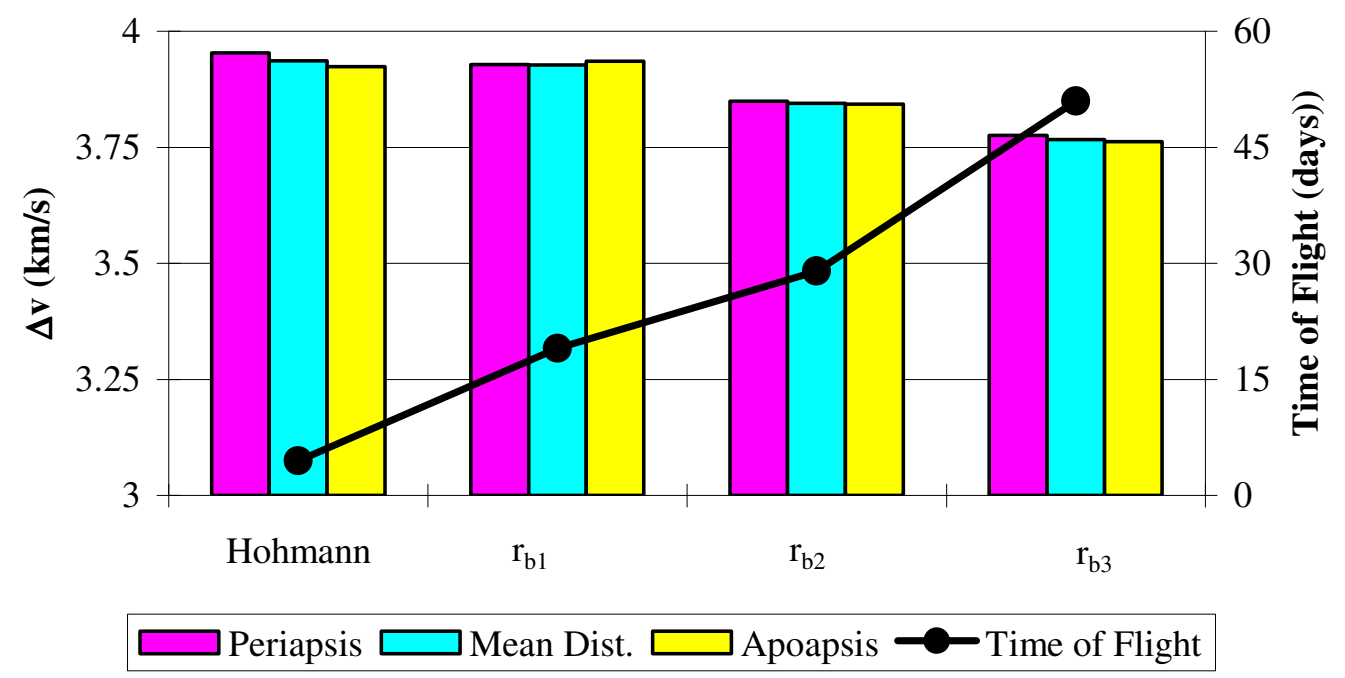

Figure 12 Comparing Hohmann and Bi-Elliptic $\Delta v$ Requirements and TOF 
Significant $\Delta \mathrm{v}$ savings using the bi-elliptic is achieved with large intermediate orbit radius $\left(\mathrm{r}_{\mathrm{b}}\right)$ and these savings are attained at the cost of a considerably increased TOF. In addition, the savings that can be realized using bi-elliptic orbits with a reasonable TOF are likely to be within the margins that exist in a full-scale spacecraft design. The usefulness of these $\Delta v$ savings is apparent in the fuel mass, which is discussed later in Section 3.2 on the CRS propulsion system.

\subsubsection{Patched Conic}

The Hohmann and bi-elliptic methods provide a useful first approximation of the $\Delta v$ needed for lunar transfer. The patched conic method is used to refine this estimate [17]. This refinement uses the same two-body mechanics as the Hohmann; however, it accounts for effects of the Moon's gravity by using a concept known as the sphere of influence (SOI). This construct observes that at a certain distance between two astronomical bodies the force of gravity on a spacecraft is equal from each body, and shifts the reference frame from one body to the other at this boundary. In this case, when the spacecraft reaches the boundary of the Moon's SOI, the position and velocity vectors are transformed from a coordinate system centered at the Earth to one centered at the Moon and the gravitational parameter $(\mu)$ changes to reflect the Moon as the central body for the spacecraft. This requires careful calculation of the orbital position, flight path angle, and velocity since the Moon moves relative to the Earth. Fig. 13 shows a representative patched conic transfer.

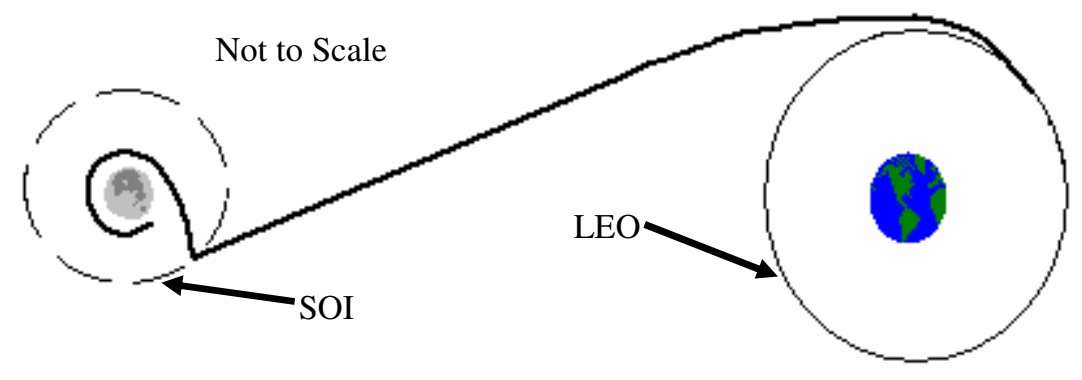

Figure 13 Patched Conic Transfer

The patched conic approximation is computed in the following manner [17]. First, the radius of the sphere of influence $\left(R_{\mathrm{s}}\right)$ is calculated using equation (11) where $D$ is the Earth-Moon average distance, and $\mu$ are the gravitational parameters of the Earth and Moon. 


$$
R_{s}=D\left(\frac{\mu_{\text {Moon }}}{\mu_{\text {Earth }}}\right)^{2 / 5}
$$

In order to determine the trajectory, several independent variables have values chosen for them. These include the departure radius $\left(r_{0}\right)$, departure velocity $\left(v_{0}\right)$, departure flight-path-angle $\left(\phi_{0}\right)$, and the angle entering the lunar SOI $\left(\lambda_{1}\right)$. The example given in Ref. 17 has the spacecraft start out in a $300 \mathrm{~km}$ altitude circular low-Earth orbit $\left(r_{0}=6678 \mathrm{~km}\right)$. It is given a boost from its initial orbital velocity of $7.72 \mathrm{~km} / \mathrm{s}$ to the departure velocity $\left(v_{0}\right)$ of 10.84 $\mathrm{km} / \mathrm{s}(\Delta \mathrm{v}$ of $3.78 \mathrm{~km} / \mathrm{s})$. The maneuver is done with a flight path angle $\left(\phi_{0}\right)$ of $0^{\circ}$ and an angle entering the lunar SOI $\left(\lambda_{1}\right)$ of $30^{\circ}$. The transfer orbit energy $(\xi)$ and transfer orbit angular momentum $(h)$ are computed from equations (12-13).

$$
\begin{gathered}
\xi=\frac{v_{0}^{2}}{2}-\frac{\mu_{E a r t h}}{r_{0}} \\
h=r_{0} v_{0} \cos \phi_{0}
\end{gathered}
$$

The orbital radius $\left(r_{l}\right)$ and the orbital velocity $\left(v_{l}\right)$ upon arrival at the lunar SOI are computed from equations (14$15)$.

$$
\begin{gathered}
r_{1}=\sqrt{D^{2}+R_{s}^{2}-2 D R_{s} \cos \lambda_{1}} \\
v_{1}=\sqrt{2\left(\xi+\mu_{E a r t h} / r_{1}\right)}
\end{gathered}
$$

A check is performed to ensure that $v_{l}$ contains only real quantities. If it does not, the transfer orbit does not have enough energy to reach the Moon's SOI due to the choice of initial parameters. An adjustment in these initial parameters must be done. The remaining arrival conditions, the flight-path-angle $\left(\phi_{0}\right)$, and phase angle at arrival $\left(\gamma_{1}\right)$ which is the angle subtended by the Earth-Moon position vector and the Earth-CRS position vector are computed with equations (16-17).

$$
\begin{gathered}
\phi_{1}=\cos ^{-1}\left(\frac{h}{r_{1} v_{1}}\right) \\
\gamma_{1}=\sin ^{-1}\left(\frac{R_{s}}{r_{1}} \sin \lambda_{1}\right)
\end{gathered}
$$


To determine the time-of-flight for the transfer trajectory, the parameter $(p)$, the semi-major axis $(a)$, and the eccentricity $(e)$ of the transfer orbit are found using equations (18-20).

$$
\begin{gathered}
p=\frac{h^{2}}{\mu_{\text {Earth }}} \\
a=-\frac{\mu_{\text {Earth }}}{2 \xi} \\
e=\sqrt{1-p / a}
\end{gathered}
$$

Using the true anomalies at departure $\left(v_{0}\right)$ and arrival $\left(v_{1}\right)$ from equations (21-22) and the eccentric anomalies at departure $\left(E_{0}\right)$ and arrival $\left(E_{1}\right)$ from equations (23-24), the time-of-flight is calculated from equation (25).

$$
\begin{gathered}
v_{0}=\cos ^{-1}\left(\frac{p-r_{0}}{e r_{0}}\right) \\
v_{1}=\cos ^{-1}\left(\frac{p-r_{1}}{e r_{1}}\right) \\
E_{0}=\tan ^{-1}\left[\frac{\left(\frac{\sqrt{1-e^{2}} \sin v_{0}}{1+e \cos v_{0}}\right)}{\left(\frac{e+\cos v_{0}}{1+e \cos v_{0}}\right)}\right] \\
E_{1}=\tan ^{-1}\left[\frac{\left(\frac{\sqrt{1-e^{2}} \sin v_{1}}{1+e \cos v_{1}}\right)}{\left(\frac{e+\cos v_{1}}{1+e \cos v_{1}}\right)}\right] \\
\text { TOF }=\sqrt{\frac{a^{3}}{\mu_{E a r t h}}}\left[\left(E_{1}-e \sin E_{1}\right)-\left(E_{0}-e \sin E_{0}\right)\right]
\end{gathered}
$$

Another check is performed to ensure that the trajectory is possible. This is done by computing the phase angle with equation (26). Note that the angular velocity of the Moon about the Earth is $\omega_{m}=2.649 \times 10^{-6}$ radians per second in this equation. 


$$
\gamma_{0}=v_{1}-v_{0}-\omega_{m} \times T O F
$$

A series of critical steps follow as calculations are completed to analyze the conditions at the patch point. Fig. 14 shows the angular relationships at the patch point [17].

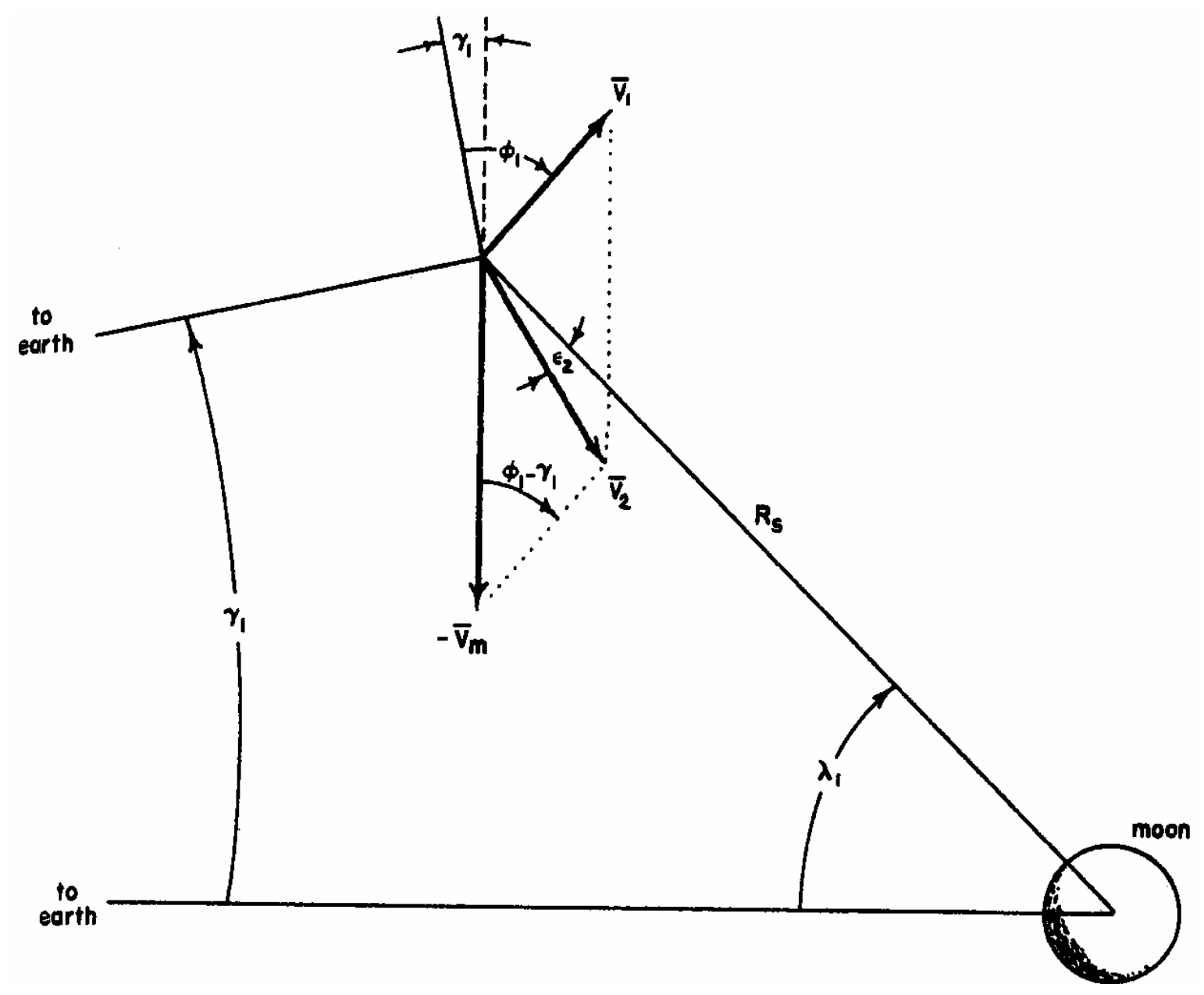

Figure 14 Conditions at the Patch Point

The lunar arrival speed is found by applying the law of cosines as in equation (27). Note the velocity of the Moon about the Earth used for this calculation is $v_{m}=1.018 \mathrm{~km} / \mathrm{s}$.

$$
v_{2}=\sqrt{v_{1}^{2}+v_{m}^{2}-2 v_{1} v_{m} \cos \left(\phi_{1}-\gamma_{1}\right)}
$$

The angle $\left(\varepsilon_{2}\right)$ which defines the velocity vector of the CRS relative to the Moon's center is computed using equation (28). 


$$
\varepsilon_{2}=\sin ^{-1}\left(\frac{v_{m}}{v_{2}} \cos \lambda_{1}-\frac{v_{1}}{v_{2}} \cos \left(\lambda_{1}+\gamma_{1}-\phi_{1}\right)\right)
$$

The next several equations determine the orbit of the CRS inside the lunar SOI. The subscripts of two indicate the orbital parameters as distinct from the initial and transfer orbits. Orbit energy $\left(\xi_{2}\right)$, angular momentum $\left(h_{2}\right)$, parameter $\left(p_{2}\right)$, and eccentricity $\left(e_{2}\right)$ are computed from equations (29-32). Note that, $r_{2}=R_{s}$ which states that the satellite position vector is equal in magnitude to the radius of the SOI.

$$
\begin{gathered}
\xi_{2}=\frac{v_{2}^{2}}{2}-\frac{\mu_{M o o n}}{r_{2}} \\
h_{2}=r_{2} v_{2} \sin \varepsilon_{2} \\
p_{2}=\frac{h_{2}^{2}}{\mu_{M o o n}} \\
e_{2}=\sqrt{1+2 \xi_{2} h_{2}^{2} / \mu_{M o o n}^{2}}
\end{gathered}
$$

The point of closet approach to the Moon $\left(r_{\mathrm{p}}\right)$ and the velocity at this point $\left(v_{\mathrm{p}}\right)$ can be found from equations (33-34).

$$
\begin{gathered}
r_{p}=\frac{p_{2}}{1+e_{2}} \\
v_{p}=\sqrt{2\left(\xi_{2}+\mu_{\text {Moon }} / r_{p}\right)}
\end{gathered}
$$

Note that the radius of the Moon $\left(\mathrm{R}_{\mathrm{m}}\right)$ is $1738 \mathrm{~km}$. A check is performed to ensure that $r_{p}$ is greater than this radius.

Additional calculations can be performed to find the TOF inside the lunar SOI. The semi-major axis of the new orbit is found using equation (35).

$$
a_{2}=\frac{\mu_{M o o n}}{2 \xi_{2}}
$$

Equations (21-25) are reapplied to find the TOF. The total TOF is found by summing the earlier value from equation (25) and this result.

Since the arrival orbit is hyperbolic, a $\Delta \mathrm{v}$ can be performed to enter an orbit around the Moon. For this calculation, the $\Delta \mathrm{v}$ is performed at the perilune of the arrival orbit. An orbit eccentricity $\left(e_{3}\right)$ is chosen. The desired semi-major axis $\left(a_{3}\right)$ for the orbit is computed using equation (36). 


$$
a_{3}=\frac{r_{p}}{1-e_{3}}
$$

The velocity at the perilune $\left(v_{3}\right)$ is computed from equation (37).

$$
v_{3}=\sqrt{\frac{2 \mu_{M o o n}}{r_{p}}-\frac{\mu_{M o o n}}{a_{3}}}
$$

The velocity change is found from equation (38).

$$
\Delta \mathrm{v}=v_{p}-v_{3}
$$

From the example, the spacecraft proceeds inside the Moon's SOI on a hyperbolic trajectory and passes the Moon at a distance of 2,367 $\mathrm{km}$ from the surface. To apply this method to the specific mission, the portion of the trajectory that is in the Earth's SOI is optimized. This is accomplished by calculating the minimum energy approach trajectory to the Moon's SOI in order to use a Hohmann transfer at the edge of the Moon's SOI. Thus the orbit's apogee just reaches the Moon's SOI, and $\lambda_{1}$ changes from $30^{\circ}$ to $0^{\circ}$. The minimum $v_{0}$ for this transfer is $10.81 \mathrm{~km} / \mathrm{s}$. With an initial orbital velocity about the Earth of $7.71 \mathrm{~km} /$, the necessary $\Delta \mathrm{v}$ is $3.09 \mathrm{~km} / \mathrm{s}$. Results for other lunar distances appear in Table 4.

\section{Table 4 Minimum Energy Earth Departure for CRS to Reach Moon SOI}

\begin{tabular}{|l|c|c|c|}
\cline { 2 - 4 } \multicolumn{1}{c|}{} & Perigee Distance & $\begin{array}{c}\text { Mean } \\
\text { Distance }\end{array}$ & $\begin{array}{c}\text { Apogee } \\
\text { Distance }\end{array}$ \\
\hline Departure Velocity $(\mathrm{km} / \mathrm{s})$ & 10.80 & 10.81 & 10.81 \\
\hline$\Delta \mathrm{v}(\mathrm{km} / \mathrm{s})$ & 3.08 & 3.09 & 3.10 \\
\hline
\end{tabular}

However, through some iteration and graphical analysis, it is found that by increasing $v_{0}$ to $10.84 \mathrm{~km} / \mathrm{s}$ and changing $\lambda_{1}$ to $58^{\circ}$ while retaining the other initial conditions, namely $r_{0}=6678 \mathrm{~km}$ and $\phi_{0}=0^{\circ}$, the CRS can approach the Moon closely. The variation of $\lambda_{1}$ is accomplished through timing of the arrival at the SOI. Details are left to a specific future design. Lunar orbit insertion (LOI) into an LLPO with a small $r_{\mathrm{p}}$ and an eccentricity of 0.1 can be achieved if a burn is done. Typically, the point of closest approach to the Moon is the best place to do the burn. This allows a single burn to satisfy most of the LLPO parameters and simplifies the design. Other eccentricities are possible and those greater than 0.1 require less $\Delta \mathrm{v}$ for LOI. The eccentricity of the lunar orbit affects the communication link as will be described in Section 3.1 and the viewing time as described earlier. It is also desirable to have perilune fairly close to the Moon, at an altitude of several hundred kilometers. Two trajectories are possible 
for lunar orbit entry. These trajectories use different angles $\left(\lambda_{1}\right)$ to enter the lunar SOI. To account for the additional flight time that occurs within the lunar SOI the method in Ref. 17 is adapted by applying the same method for determining the TOF from the Earth to the lunar SOI, but with a new central body. Computer codes that calculate the patched conic trajectory are in Appendix B. The significant transfer parameters appear in Table 5.

Table 5 Summary of Patched Conic Conditions

\begin{tabular}{|c|c|c|c|c|c|c|c|}
\hline & & \multicolumn{2}{|c|}{ Perigee Distance } & \multicolumn{2}{|c|}{ Mean Distance } & \multicolumn{2}{|c|}{$\begin{array}{l}\text { Apogee } \\
\text { Distance }\end{array}$} \\
\hline \multirow{4}{*}{ Inputs } & Orbit Radius at Earth $\left(\mathrm{r}_{0}\right)(\mathrm{km})$ & \multicolumn{2}{|c|}{6678} & \multicolumn{2}{|c|}{6678} & \multicolumn{2}{|c|}{6678} \\
\hline & $\begin{array}{l}\text { Departure Velocity from Earth }\left(\mathrm{v}_{0}\right) \\
(\mathrm{km} / \mathrm{s})\end{array}$ & \multicolumn{2}{|c|}{10.84} & \multicolumn{2}{|c|}{10.84} & \multicolumn{2}{|c|}{10.84} \\
\hline & Initial Flight Path Angle $\left(\phi_{0}\right)$ & \multicolumn{2}{|c|}{$0^{\circ}$} & \multicolumn{2}{|c|}{$0^{\circ}$} & \multicolumn{2}{|c|}{$0^{\circ}$} \\
\hline & Angle Entering Lunar SOI $\left(\lambda_{1}\right)$ & $37^{\circ}$ & $48^{\circ}$ & $45^{\circ}$ & $58^{\circ}$ & $54^{\circ}$ & $70^{\circ}$ \\
\hline \multirow{5}{*}{ Outputs } & Closest Approach to Moon (km) & 499 & 368 & 267 & 659 & 82 & 306 \\
\hline & TOF (days) & 2.78 & 2.93 & 3.38 & 3.65 & 4.05 & 4.56 \\
\hline & Earth Departure $\Delta \mathrm{v}(\mathrm{km} / \mathrm{s})$ & 3.13 & 3.13 & 3.13 & 3.13 & 3.13 & 3.13 \\
\hline & LOI $\Delta \mathrm{v}(\mathrm{km} / \mathrm{s})$ & 0.75 & 0.74 & 0.73 & 0.68 & 0.73 & 0.69 \\
\hline & Total $\Delta \mathrm{v}(\mathrm{km} / \mathrm{s})$ & 3.88 & 3.87 & 3.83 & 3.81 & 3.86 & 3.80 \\
\hline
\end{tabular}

The results verify the Hohmann transfer method since the total required $\Delta v$ is within the same range. An LLPO that resembles those discussed in Section 2.2 on mission orbits can be achieved.

Furthermore, these calculations are in reasonable agreement with the calculations in the literature. A literature summary along with comparison to the calculations appears in Figure 15.

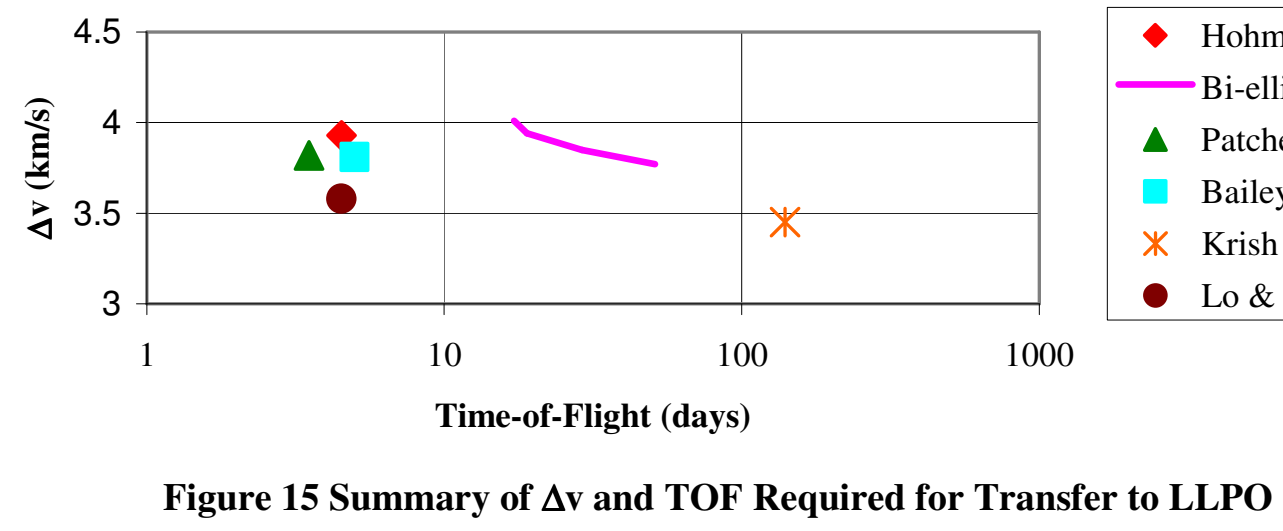

In some cases, specialized trajectories are possible which save $\Delta \mathrm{v}$ at a cost of significantly increased TOF [19]. As a reference, the Apollo 11 flight took 3.05 days to travel to the Moon and a total $\Delta \mathrm{v}$ of $3.93 \mathrm{~km} / \mathrm{s}$ for trans- 
lunar insertion and lunar orbit insertion burns [21]. Apollo 17 took 3.46 days to travel to the Moon and a total $\Delta \mathrm{v}$ of $3.95 \mathrm{~km} / \mathrm{s}$ for trans-lunar insertion and lunar orbit insertion burns [22].

\subsubsection{Non-Coplanar Lunar Trajectory}

All the prior calculations assume that the orbits lie in the same plane. The next refinement removes this assumption and analyzes a non-coplanar lunar trajectory [17]. The launch time, launch azimuth, intercept time, and intercept position of the Moon are calculated. With this method, the transfer orbit is divided into three phases, a launch phase, a LEO coasting phase, and the lunar transfer phase. There are several free parameters to be defined, thus iteration is done to refine the transfer and permit a lunar intercept, by having the CRS arrive at a point in space and time which coincides with that of the Moon. The 3D transfer calculations to the Moon more closely resemble a Hohmann transfer than a patched conic because the orbit-altering effects of the Moon's gravity are not taken into account. For this non-coplanar lunar trajectory, the injection conditions into a lunar transfer orbit are the same as for the patched conic.

Several constraints are placed on the results. The relative timing of the mission is set by a requirement to arrive at the Moon several days before new Moon. Additionally a launch that allows the CRS to intercept the Moon close to either its perigee or apogee allows for minimum transfer $\Delta \mathrm{v}$ or communication power. To meet the requirements of the AO the launch should occur in either early December 2009 or early June 2010. A launch from Cape Canaveral $\left(28.5^{\circ} \mathrm{N} 80.5^{\circ} \mathrm{W}\right)$ is assumed. Range safety constrains the launch azimuth to between $40^{\circ}$ and $115^{\circ}$ so that the launch vehicle heads out over the open waters of the Atlantic Ocean and not over populated land areas [17]. Fig. 16 shows the angular relationships of the lunar intercept [17]. 


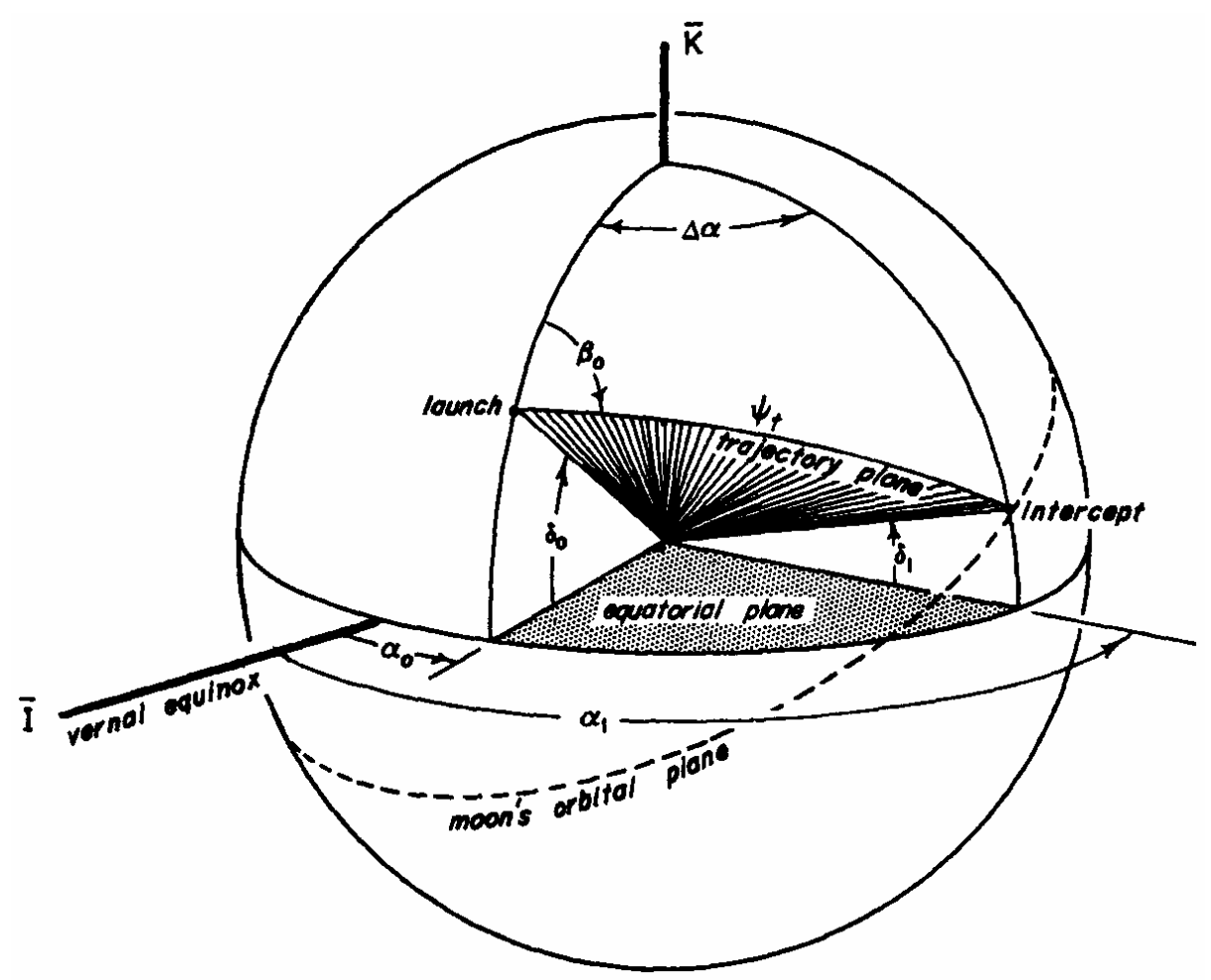

Figure 16 Angular Relationships of the Non-Coplanar Lunar Trajectory

To determine the non-coplanar lunar trajectory a two-pronged approach to this problem is followed [17].

First, the launch azimuth constraints are satisfied and then an acceptable launch time is determined such that the spacecraft reaches the Moon at the desired intercept time. With a computer code written in Matlab, it is nearly possible to satisfy all constraints simultaneously. The method entails satisfying geometric as well as timing constraints. The geometry of the launch angle, point of launch, and the point of intercept need to fit with the spherical geometry. The difference in right ascension $(\Delta \alpha)$ determines the size of the trajectory geometrically. The right ascension of the launch site $\left(\alpha_{0}\right)$ and the right ascension of the Moon at intercept $\left(\alpha_{1}\right)$ are subtracted to determine the fit from the position of the Earth and Moon. If the difference $\Delta \alpha-\left(\alpha_{1}-\alpha_{0}\right)$ is small, an intercept will occur. The date of launch is determined by subtracting the travel time from the intercept time. The travel time depends upon the fit of spherical geometry to satisfy all constraints. The entire method, with portions adapted from Ref.12 and Ref.17, appears below.

First, note that all subscripts of zero indicate at launch and all subscripts of one indicate at intercept. Also, the gravitational parameter of the Earth is $\mu\left(398600.4415 \mathrm{~km}^{3} / \mathrm{s}^{2}\right)$. 
Inputs to the calculation are the intercept time $\left(t_{l}\right)$ in year, month, day, hour, minute, and second which should be converted to Julian days, injection orbit radius in $\mathrm{km}\left(r_{0}\right)$, the injection velocity in $\mathrm{km} / \mathrm{s}\left(v_{0}\right)$, the injection flight path angle in degrees $\left(\phi_{0}\right)$, the latitude of launch site in degrees $\left(\delta_{0}\right)$, and the longitude of launch site in degrees $(\lambda)$

The right ascension $\left(\alpha_{\mathrm{h}}, \alpha_{\mathrm{m}}, \alpha_{\mathrm{s}}\right)$ of the Moon at the intercept time in hours, minutes, seconds is found. The declination $\left(\delta_{\mathrm{deg}}, \delta_{\mathrm{m}}, \delta_{\mathrm{s}}\right)$ of the Moon at the intercept time in degrees, minutes, seconds is found. The distance of the Moon $\left(r_{l}\right)$ at the intercept time is found. A useful resource for finding these quantities is an ephemeris calculator [23].

Right ascension $\left(\alpha_{1}\right)$ is converted from hour, minute, second to radians via equation (39).

$$
\alpha_{l}=15 *\left(\alpha_{\mathrm{h}}+\alpha_{\mathrm{m}} / 60+\alpha_{\mathrm{s}} / 3600\right) * \pi / 180
$$

Declination is converted from degrees, minutes, and seconds to radians via equation (40).

$$
\delta_{l}=\left(\delta_{\mathrm{deg}}+\delta_{\mathrm{m}} / 60+\delta_{\mathrm{s}} / 3600\right) * \pi / 180
$$

Equations (41-50) are similar to those used in the patched conic discussion and are repeated here for continuity. The spacecraft energy's is determined from equation (41).

$$
\xi=\frac{v_{0}^{2}}{2}-\frac{\mu}{r_{0}}
$$

The transfer orbit semi-major axis $(a)$ is found from equation (42).

$$
a=-\frac{\mu}{2 \xi}
$$

The orbit's angular momentum $(h)$ is found from equation (43).

$$
h=r_{0} v_{0} \cos \phi_{0}
$$

The orbit's parameter ( $p$ ) is found from equation (44).

$$
p=\frac{h^{2}}{\mu}
$$

The orbit's eccentricity $(e)$ is found from equation (45).

$$
e=\sqrt{1-\frac{p}{a}}
$$


The true anomaly at intercept $\left(v_{1}\right)$ and launch $\left(v_{0}\right)$ are found from equations $(46-47)$.

$$
\begin{aligned}
& v_{1}=\cos ^{-1}\left(\frac{p-r_{1}}{e r_{1}}\right) \\
& v_{0}=\cos ^{-1}\left(\frac{p-r_{0}}{e r_{0}}\right)
\end{aligned}
$$

The eccentric anomaly at intercept $\left(E_{l}\right)$ and launch $\left(E_{0}\right)$ are found from equations (48-49).

$$
\begin{aligned}
& E_{1}=\tan ^{-1}\left[\frac{\left(\frac{\sqrt{1-e^{2}} \sin v_{1}}{1+e \cos v_{1}}\right)}{\left(\frac{e+\cos v_{1}}{1+e \cos v_{1}}\right)}\right] \\
& E_{0}=\tan ^{-1}\left[\frac{\left(\frac{\sqrt{1-e^{2}} \sin v_{0}}{1+e \cos v_{0}}\right)}{\left(\frac{e+\cos v_{0}}{1+e \cos v_{0}}\right)}\right]
\end{aligned}
$$

The free-flight time $\left(t_{f f}\right)$ and angle $\left(\psi_{f f}\right)$ are found using equations (50-51).

$$
\begin{gathered}
t_{f f}=\sqrt{\frac{a^{3}}{\mu}}\left[\left(E_{1}-e \sin E_{1}\right)-\left(E_{0}-e \sin E_{0}\right)\right] \\
\psi_{f f}=v_{1}-v_{0}
\end{gathered}
$$

The free-flight time $\left(t_{f f}\right)$ is calculated with all angular quantities in radians. The coasting angle $\left(\psi_{c}\right)$ requires an initial estimate. Discussion on refinement follows. The total sweep angle $\left(\psi_{t}\right)$ is found from equation (52).

$$
\psi_{t}=\psi_{f f}+\psi_{c}
$$

The launch azimuth $\left(\beta_{0}\right)$ is found from equation (53)

$$
\cos \beta_{0}=\frac{\sin \delta_{1}-\sin \delta_{0} \cos \psi_{t}}{\cos \delta_{0} \sin \psi_{t}}
$$

The eccentric anomalies are recalculated using equations (48) and (49). For this calculation, the following values are set: $e=0, a=r_{0}, v_{0}=0$, and $v_{1}=\psi_{c}$. 
The coasting time $\left(t_{c}\right)$ is found from equation (54) using the recalculated eccentric anomalies.

$$
t_{c}=\sqrt{\frac{a^{3}}{\mu}}\left[\left(E_{1}-e \sin E_{1}\right)-\left(E_{0}-e \sin E_{0}\right)\right]
$$

The launch time $\left(t_{0}\right)$ is found from equation (55).

$$
t_{0}=t_{1}-\left(t_{c}+t_{f f}\right)
$$

As mentioned earlier, the launch time is best computed using Julian Days in all quantities and then the output reconverted to a standard form of mmm.dd,yyyy HH:MM:SS for easier viewing. The right ascension of the launch point $\left(\alpha_{0}\right)$ is found using the launch time $\left(t_{0}\right)$ and the launch longitude $(\lambda)$ via equations (56-57).

$$
\begin{gathered}
T_{U T 1}=\frac{t_{0}-2451545}{36525} \\
\alpha_{0}=67310.54841^{\mathrm{s}}+\left(876600^{\mathrm{h}}+8640184.812866^{\mathrm{s}}\right) \mathrm{T}_{\mathrm{ut} 1} \\
+.093104 \mathrm{~T}_{\mathrm{ut} 1}^{2}-6.2 \times 10^{-6} \mathrm{~T}_{\mathrm{ut} 1}^{3}+\lambda
\end{gathered}
$$

The geometric difference in right ascension between launch and intercept is found using equation (58).

$$
\cos \Delta \alpha=\frac{\cos \psi_{t}-\sin \delta_{0} \sin \delta_{1}}{\cos \delta_{0} \cos \delta_{1}}
$$

The geometric difference $\Delta \alpha$ is compared to the time-calculated right ascension difference $\alpha_{1}-\alpha_{0}$. If $\Delta \alpha-\left(\alpha_{1}-\alpha_{0}\right)$ is within a desirable tolerance ( $1^{\circ}$ was deemed acceptable for this method), the launch azimuth is checked to see that it is within the desired safety range (Cape Canaveral is $40^{\circ}$ up to $115^{\circ}$ ). If the launch azimuth is within the tolerance, the algorithm is complete. If $\Delta \alpha-\left(\alpha_{l^{-}} \alpha_{0}\right)$ is not acceptable, the coasting angle $\left(\psi_{c}\right)$ is adjusted until it is. If an acceptable difference cannot be found or if an acceptable launch azimuth cannot be found, a new intercept date is chosen. It is suggested to move the intercept date several hours in advance or behind the previously chosen date. To determine a launch window, the injection velocity must be adjusted and then the coasting angle varied until the launch azimuth is within constraints and the difference $\Delta \alpha-\left(\alpha_{1}-\alpha_{0}\right)$ is acceptable.

A Matlab computer code that performs this calculation appears in Appendix B. Results from the calculation appear in Table 6. 


\section{Table 6 Non-Coplanar Lunar Trajectory Conditions Launch Opportunities}

\begin{tabular}{|c|c|c|}
\hline & First Opportunity & Second Opportunity \\
\hline Launch Date/Time $\left(t_{0}\right)$ & Dec.05, 2009 19:51:36 UTC & Jun.01, 2010 01:43:22 UTC \\
\hline Launch Azimuth $\left(\beta_{0}\right)$ & $40.16^{\circ}$ & $82.31^{\circ}$ \\
\hline Right Ascension of Launch Site $\left(\alpha_{0}\right)$ & Oh $44 \mathrm{~m} 56.588 \mathrm{~s}$ & $18 \mathrm{~h} 15 \mathrm{~m} 30.674 \mathrm{~s}$ \\
\hline Intercept Date/Time $\left(t_{1}\right)$ & Dec.09, 2009 06:00:00 UTC & Jun.05, 2010 08:00:00 UTC \\
\hline $\begin{array}{l}\text { Moon Right Ascension at Intercept } \\
\left(\alpha_{1}\right)\end{array}$ & $11 \mathrm{~h} 17 \mathrm{~m} \mathrm{39.247s}$ & $23 \mathrm{~h} 12 \mathrm{~m} 11.534 \mathrm{~s}$ \\
\hline Moon Declination at Intercept $\left(\delta_{1}\right)$ & $-0^{\circ} 17^{\prime} 23.18^{\prime \prime}$ & $0^{\circ} 5^{\prime} 31.74^{\prime \prime}$ \\
\hline Moon Dist. at Intercept $\left(r_{1}\right)$ & 58.714 Earth Radii & 63.118 Earth Radii \\
\hline Coasting Angle $\left(\psi_{\mathrm{c}}\right)$ & $330^{\circ}$ & $107^{\circ}$ \\
\hline Coasting Time $\left(t_{\mathrm{c}}\right)$ & 0.05 days & 0.01 days \\
\hline Free-Flight Angle $\left(\psi_{\mathrm{ff}}\right)$ & $174.95^{\circ}$ & $177.03^{\circ}$ \\
\hline Free-Flight Time $\left(t_{\mathrm{ff}}\right)$ & 3.36 days & 4.24 days \\
\hline$\Delta \alpha$ & $158.26^{\circ}$ & 74.038 \\
\hline$\Delta \alpha-\left(\alpha_{1}-\alpha_{0}\right)$ & $0.08^{\circ}$ & $-0.13^{\circ}$ \\
\hline
\end{tabular}

For each of these opportunities, it is estimated that a launch window of approximately seven hours exists. This time is limited by the range safety constraints. The method demonstrates that it is possible to find a non-coplanar lunar trajectory that satisfies the constraints without too much difficulty.

\subsubsection{Transfers to $L_{2}$}

The second Lagrange point in the Earth-Moon system is an unstable saddle point located on average 64,500 km directly behind the Moon as viewed from the Earth. The position of the point is determined by the orbital and gravitational mechanics of the Moon and Earth, and is a fixed fraction of the changing distance between the two bodies.

Initially, Hohmann transfers from LEO to an orbit at this distance from the Earth are considered. However, because a spacecraft does not have the same velocity at the $\mathrm{L}_{2}$ point as it does for a standard 2-body case, it is necessary to augment the spacecraft velocity so that it orbits the Earth-Moon barycenter with the same period as the Moon orbits the Earth, but at a greater distance. The precise details of entering a halo orbit are left to a full-scale design. 
As with transfers directly to the Moon, Hohmann transfers and bi-elliptic transfers provide the required $\Delta \mathrm{v}$. The standard equations for calculating the Hohmann transfer are used as was discussed in Section 2.3.1. The CRS departs LEO from a $300 \mathrm{~km}$ altitude orbit. Then additional $\Delta \mathrm{v}$ is necessary at $\mathrm{L}_{2}$. The $\Delta \mathrm{v}$ and TOF requirements for the $\mathrm{L}_{2}$ point at different distances are calculated. For the bi-elliptic case, the same equations are used, but the CRS first transfers into an elliptical orbit with apogee at a radius $\left(\mathrm{r}_{\mathrm{b}}\right)$ greater than the final radius, then into a second elliptical orbit, from which it transfers to the final circular orbit. This method increases the TOF, but requires less $\Delta v$ for most cases. Three different intermediate radii are chosen and the results appear in Figure 17. The intermediate radii are: $\mathrm{r}_{\mathrm{b} 1}=4 \times 10^{5} \mathrm{~km}, \mathrm{r}_{\mathrm{b} 2}=6 \times 10^{5} \mathrm{~km}, \mathrm{r}_{\mathrm{b} 3}=1 \times 10^{6} \mathrm{~km}$.

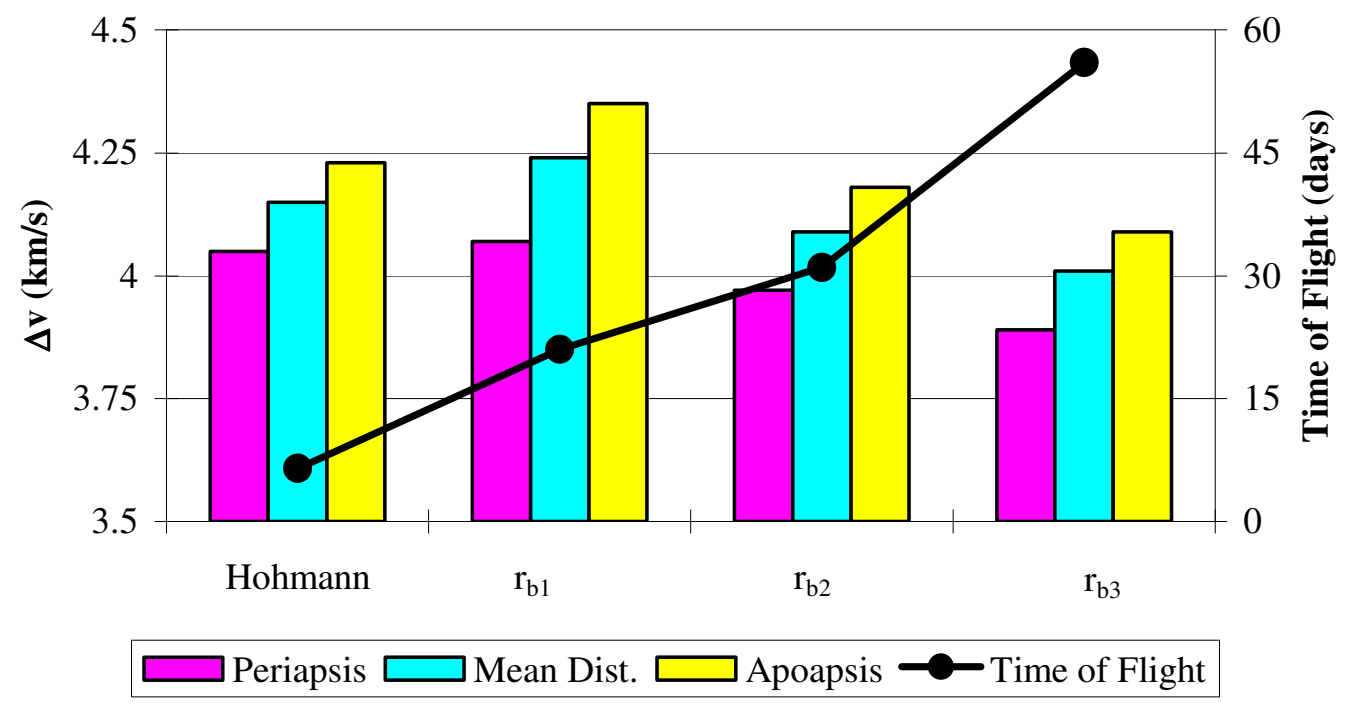

Figure $17 \Delta v$ and TOF Requirements for Hohmann or Bi-Elliptic $L_{2}$ Transfer

There is not a significant $\Delta v$ savings until the intermediate orbit radius $\left(\mathrm{r}_{\mathrm{b}}\right)$ is large. Once this occurs, the TOF has become long and even then the fuel savings that can be realized using bi-elliptic orbits are likely to be within the margins that exist in a full-scale spacecraft design.

To get a more precise estimate of the $\Delta \mathrm{v}$, an examination of the literature was done. Farquhar shows a twoimpulse transfer that can be accomplished with $4.42 \mathrm{~km} / \mathrm{s}$ and a transfer time of four days [13]. A slightly more complicated three-impulse transfer, which includes a near pass of the Moon, can be accomplished with $3.53 \mathrm{~km} / \mathrm{s}$ and a total transfer time of nearly nine days. This $\Delta v$ requirement is less than the amount necessary to reach LLPO [13]. In Figure 18, the previous calculations are compared to the literature. 


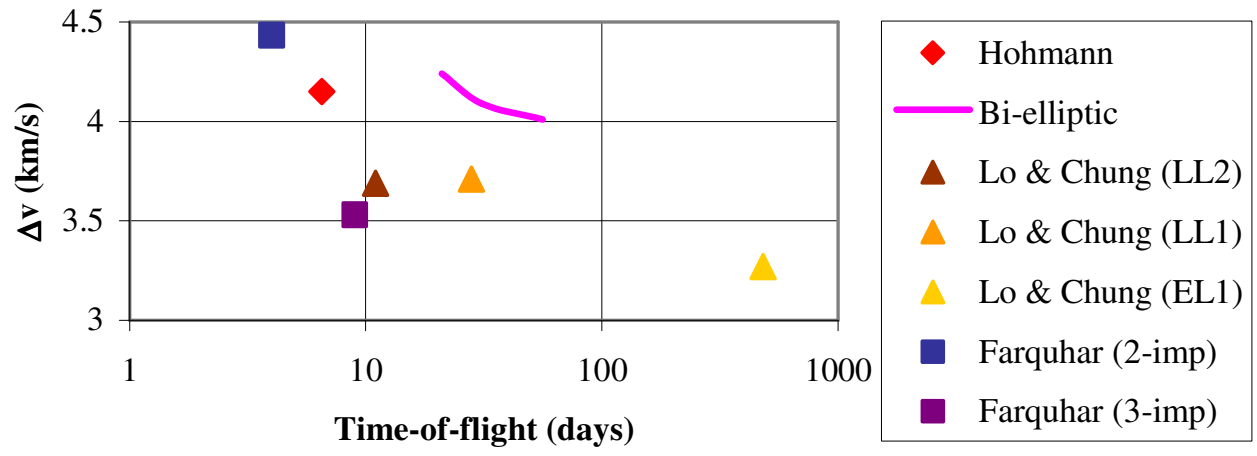

\section{Figure 18 Summary of $\Delta v$ and TOF Requirements for Transfer to $L_{2}$}

Additional $\Delta \mathrm{v}$ is required to place the CRS in a halo orbit about the $\mathrm{L}_{2}$ point. In a full-scale design, the CRS would not necessarily go directly to the $\mathrm{L}_{2}$ point. Rather a series of rocket burns are performed along the transfer trajectory to enter the halo orbit. However, if the CRS is at the $\mathrm{L}_{2}$ point, several burns are necessary to enter the halo orbit and to remain there. Farquhar notes: "Although the $\Delta \mathrm{v}$ requirements were calculated for trajectories terminating at $\mathrm{L}_{2}$, they closely approximate the corresponding values for transfers to small-amplitude halo orbits around $\mathrm{L}_{2}$ " [13]. In all cases of Lo and Chung, they took into account the insignificant amount of $\Delta \mathrm{v}$ to place the CRS into a halo orbit about $\mathrm{L}_{2}[20]$. 


\section{CRS Design}

The spacecraft contains a number of systems, whose design varies depending on the precise mission scenario (LLPO vs. $\mathrm{L}_{2}$ ). These systems included communications, propulsion, and power. Several other systems will be discussed, but their design would remain largely the same regardless of mission type including attitude determination and control, thermal, onboard computing designs, and structure.

\subsection{Communications}

The communications system provides the means of relaying data about mission critical events from the lander to Earth and for commands to be sent to the lander. It also allows the return of CRS health and housekeeping data and other information to Earth.

There are four different communication links to be made: (1) commands from the Earth to the CRS, (2) commands from the CRS to the lander, (3) data from the lander to the CRS, and (4) data from the CRS to Earth. The communications link was quantified by link budgets. These are provided by other sources and are used to size the antennas, select data rates, and estimate power requirements for the CRS's communications system [16, 24]. Some assumptions are made concerning the antenna and power output capabilities of both the lander and the Earth. NASA's Deep Space Network (DSN) has antennas with extremely high gains and output power to support the links to and from Earth [16]. The $34 \mathrm{~m}$ antenna is assumed to be available and have a gain of about 118 to $119 \mathrm{dBi}$ based on the communications frequency and dish diameter and shape [16]. It is assumed to have enough power to send commands to the CRS and receive data. Both the lander and the CRS are assumed to have high gain antennas with a gain of $15 \mathrm{dBi}$ and a transmitter power output of $50 \mathrm{~W}$. The communications frequencies are S-band, $2.5 \mathrm{GHz}$ for receiving data and $2.65 \mathrm{Ghz}$ for sending commands. Some additional assumptions were made that include losses due to space, atmosphere, and electronics and a bit error rate of one in one million.

A communications budget summary appears in Tables 7 and 8 . As shown in Table 8, the CRS at $\mathrm{L}_{2}$ has a limited data rate due to the great distance between it and the lander, but this was not the case for Earth since it was assumed that the DSN has adequate power. 
Table 7 LLPO Communications Budget

\begin{tabular}{|c|c|c|c|c|}
\cline { 2 - 5 } \multicolumn{1}{c|}{} & \multicolumn{2}{c|}{ Commands } & \multicolumn{2}{c|}{ Data } \\
\cline { 2 - 5 } \multicolumn{1}{c|}{} & $\begin{array}{c}\text { Earth to } \\
\text { CRS }\end{array}$ & $\begin{array}{c}\text { CRS to } \\
\text { Lander }\end{array}$ & $\begin{array}{c}\text { Lander to } \\
\text { CRS }\end{array}$ & $\begin{array}{c}\text { CRS to } \\
\text { Earth }\end{array}$ \\
\hline Trans Power $(\mathrm{W})$ & 500 & 50 & 50 & 50 \\
\hline Trans Ant Gain $(\mathrm{dBi})$ & 119 & 15 & 15 & 15 \\
\hline Rec Ant Gain $(\mathrm{dBi})$ & 15 & 15 & 15 & 118 \\
\hline Average Range $(\mathrm{km})$ & 384,400 & 2,000 & 2,000 & 384,400 \\
\hline Data Rate $(\mathrm{kbps})$ & 2 & 2 & 2,000 & 2,000 \\
\hline Margin $(\mathrm{dB})$ & 105 & 38 & 7 & 64 \\
\hline
\end{tabular}

Table $8 \mathrm{~L}_{2}$ Communications Budget

\begin{tabular}{|c|c|c|c|c|}
\cline { 2 - 5 } \multicolumn{1}{c|}{} & \multicolumn{2}{c|}{ Commands } & \multicolumn{2}{c|}{ Data } \\
\cline { 2 - 5 } \multicolumn{1}{c|}{} & $\begin{array}{c}\text { Earth to } \\
\text { CRS }\end{array}$ & $\begin{array}{c}\text { CRS to } \\
\text { Lander }\end{array}$ & $\begin{array}{c}\text { Lander to } \\
\text { CRS }\end{array}$ & $\begin{array}{c}\text { CRS to } \\
\text { Earth }\end{array}$ \\
\hline Trans Power $(\mathrm{W})$ & 500 & 50 & 50 & 50 \\
\hline Trans Ant Gain $(\mathrm{dBi})$ & 119 & 15 & 15 & 15 \\
\hline Rec Ant Gain $(\mathrm{dBi})$ & 15 & 15 & 15 & 118 \\
\hline Average Range $(\mathrm{km})$ & 448,900 & 64,500 & 64,500 & 448,900 \\
\hline Data Rate $(\mathrm{kbps})$ & 2 & 2 & 2 & 2,000 \\
\hline Margin $(\mathrm{dB})$ & 103 & 8 & 7 & 63 \\
\hline
\end{tabular}

Any number of parameters could be altered to increase the data rate. As an example, to enable transmission in $\mathrm{L}_{2}$ at the same data rate as in LLPO (2,000 kbps), an increase in the transmitter power to an unreasonable 40,000 Watts or a total increase in receive and transmit antenna gains of $29 \mathrm{dBi}$ must be accomplished. It is impractical to increase the power to 40,000 Watts. Other combinations of increased power and gain would also maintain the margin. Two examples for $\mathrm{L}_{2}$ are given in Table 9.

Table 9 Higher Data Rates for Lander to CRS $\mathrm{L}_{2}$ Communications

\begin{tabular}{|c|c|c|}
\hline & High Power & High Gains \\
\hline Trans Power $(\mathrm{W})$ & 40,000 & 50 \\
\hline Trans Ant Gain (dBi) & 15 & 44 \\
\hline Rec Ant Gain (dBi) & 15 & 15 \\
\hline Average Range (km) & 64,500 & 64,500 \\
\hline Data Rate (kbps) & 2,000 & 2,000 \\
\hline Margin (dB) & 6 & 6 \\
\hline
\end{tabular}

Once an adequate link is assured, the mission orbits are examined for their data return capability during the epoch. Since the average distance of a particular mission orbit varies considerably, more data can be returned by 
maximizing the rate for each distance. Data rates can be increased by several orders of magnitude, whereas the viewing time can only change by a single order of magnitude among the different orbits. More data can be returned by an LLPO with an eccentricity of zero, but since mission planners may decide that continuous viewing of the lander is more important than maximizing data return, they may prefer an $\mathrm{L}_{2}$ halo orbit. The flyby orbit allows less than half of the data return of the $\mathrm{L}_{2}$ halo orbit, which is much less than the LLPO. Figure 19 summarizes the comparison. The data return for the $\mathrm{L}_{2}$ halo orbit is for the nominal case and not for high power or gains.

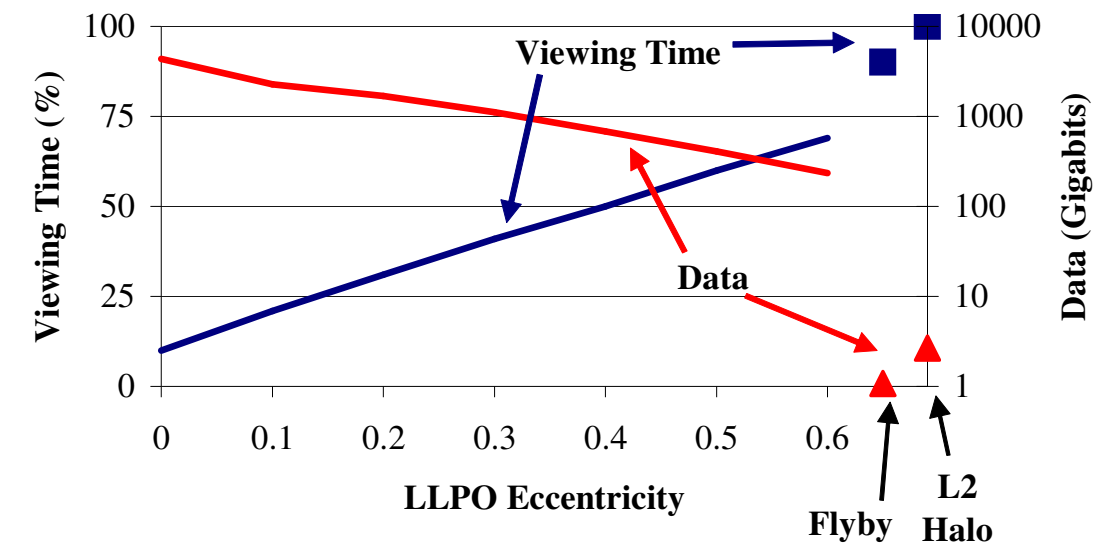

Figure 19 Data Return and Viewing Time for Mission Orbits

\subsection{Propulsion}

A propulsion system is required to provide the CRS with the necessary $\Delta \mathrm{v}$ to change and maintain orbits. It consists of a rocket, tanks, fuel, and various plumbing components. Plumbing components consist of several types of valves such as check valves, pyrotechnic valves, thruster valves, service valves, and regulator valves. Other parts include filters, tubing, gages, and sensors. A liquid bipropellant propulsion system would be useful for orbit transfer as well as orbit maintenance. A liquid propulsion system is suggested due to the efficiency and ability to throttle and restart the rocket. This capability to restart is necessary to make trajectory correction maneuvers as the spacecraft's course is refined based on tracking information. Additionally, a tank of pressurant is required to get the propellants from their tanks to the combustion chamber.

To quantify the usefulness of the $\Delta v$ savings previously discussed in Section 2.3 on transfer orbits, equation (3) is used again [16]. Here, the specific impulse, $I_{s p}$, is again assumed to be $300 \mathrm{~s}$, a range of values for $m_{f}$ is 
assumed to cover different possible CRS designs, and the range of $\Delta v$ values computed in the transfer orbits section are used. One additional fact to note is that having smaller tanks reduces the dry mass of the spacecraft.

When the $\Delta v$ requirement is reduced from a high of $4.5 \mathrm{~km} / \mathrm{s}$ to a low of $3.25 \mathrm{~km} / \mathrm{s}$, there is a fuel savings of $160 \mathrm{~kg}$ to $479 \mathrm{~kg}$ depending on the spacecraft dry mass. The savings is about $44 \%$ of the original fuel mass.

Depending on the design maturity level, anywhere from 10-25\% design margin is allocated to a spacecraft's mass [16]. This means that each given level of fuel mass has that much greater fuel mass for the initial design and these savings are still relevant. The complete fuel savings are shown in Figure 20.

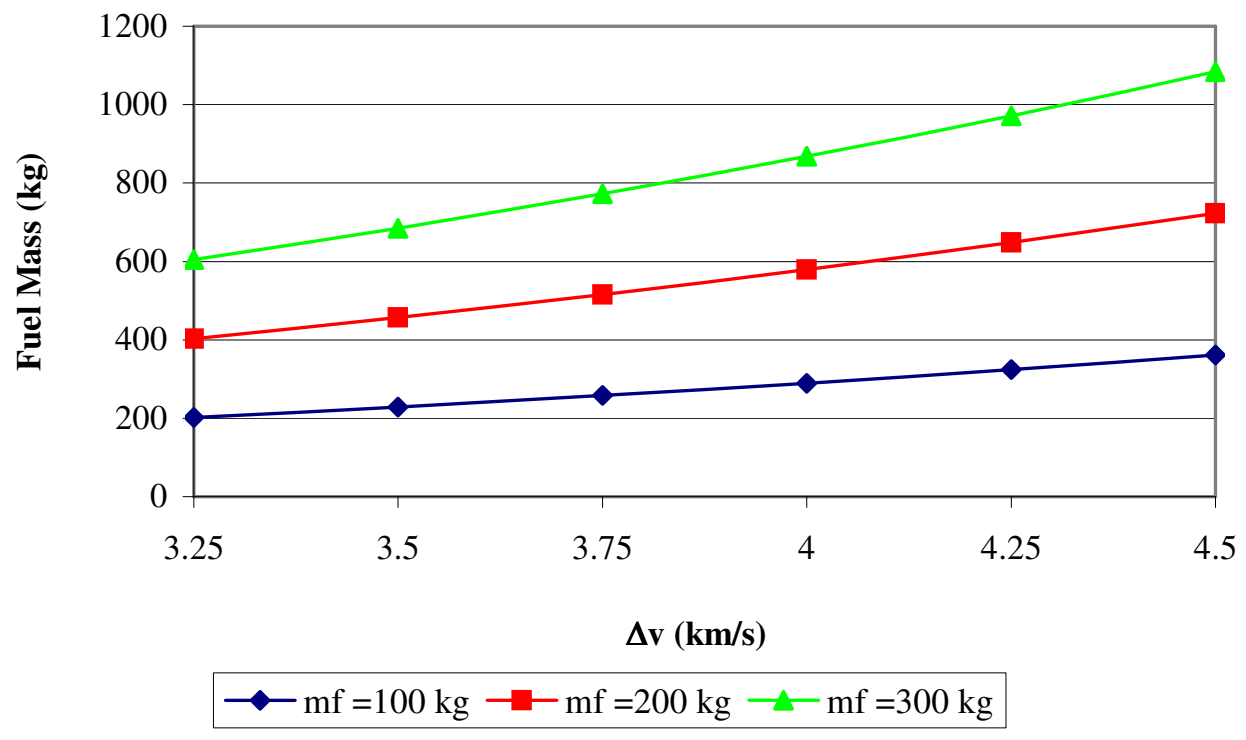

Figure 20 Fuel Mass as a Function of $\Delta v$ and Spacecraft Dry Mass

Additionally, if mission planners intend to keep the CRS at $\mathrm{L}_{2}$ or in LLPO for a long time, additional fuel is required to maintain the orbit over this period. In $\mathrm{L}_{2}$, the $\Delta \mathrm{v}$ orbit maintenance requirements can be kept as low as $23.5 \mathrm{~m} / \mathrm{s}$ per year [13]. This corresponds to 0.8 to $2.4 \mathrm{~kg}$ of propellant per year depending on CRS mass. In LLPO, some fuel is necessary to maintain orbit which is approximately the same order of magnitude as the requirement for $\mathrm{L}_{2}$, but these are not analyzed in detail.

\subsection{Power}

The CRS requires a power system that generates, stores, and distributes power within the spacecraft. The system consists of solar cells, batteries, and electronic devices to allot power to components on the CRS. The 
electronic devices include converters, cabling, fault protection, and switching gear [16]. The two different mission options require the same basic components, though in different quantities.

For LLPO, the CRS has a few solar eclipses depending on the orbit eccentricity. Lower eccentricities with the baseline mission orbit have no eclipses, but higher eccentricities have infrequent solar eclipse periods as was shown in Fig. 6. It should be noted that at high eccentricities, the Sun viewing time is slightly less than $100 \%$ due to eclipses. The CRS requires batteries to power it during solar eclipse. Communication is a high-power usage activity on the spacecraft. Since a portion of this high-usage time occurs when the spacecraft is in solar eclipse, the batteries need to provide power at high rates so that the CRS can exchange data and commands with Earth and the lander. Modern space-qualified batteries such as nickel-hydrogen and lithium-ion can meet these requirements.

For $\mathrm{L}_{2}$, there are no eclipses during the 15-day mission period. However, batteries should be included on the CRS for a number of reasons. If the CRS is configured for long-term usage, there would be brief eclipses of the Sun by the Earth and Moon on irregular occasions. If the 3-impulse transfer of Farquhar is done, then a close pass behind the Moon occurs, thus necessitating the inclusion of batteries [13]. The batteries could be significantly smaller than for the case of LLPO. This was because the CRS would not need to undertake high power operations requiring battery usage during the solar eclipse.

Solar arrays that are panel mounted are recommended since this allows the solar arrays to independently track the Sun and maximize the produced power. If a fault occurs on the spacecraft, such that for example the solar arrays were not aimed at the Sun, the batteries must provide a backup to return the spacecraft to a normal state. It is assumed that communications with Earth would occur at lower rates during this situation and adequate power would be available. Additionally, since the CRS would be limited to communicating with Earth, the lander would need to have some capability to perform autonomously. A suggestion is for the lander to remain in a safe mode for several days until the CRS can be restored. However, if the CRS was unable to be restored, the mission should consider giving the lander contingency capability whereby it should collect a rock sample and return it, absent instructions and with no sample selection interaction from Earth.

Since the primary function of the CRS is to communicate data, it is expected that the communications system uses the most power of all the systems. Propulsion requires significant power during rocket burns, but these take a small percentage of the total mission time. Systems such as attitude determination and control, thermal, and 
command and data handling require power on a continual basis to operate the CRS, but the power requirements differ little among the mission orbit options.

Power consumption is estimated for the two mission orbit options. According to Ref. 16, for a small spacecraft $(\sim 200 \mathrm{~W})$, the communications system would require about $5 \%$ of the operating power. This assumes a payload, such as science instruments or cameras, consuming $40 \%$ of the power [16]. For the CRS, the communications system is the payload and can be assumed to consume $40-45 \%$ of the power. Since the communications system uses $50 \mathrm{~W}$ when operating, the total power would be $125 \mathrm{~W}$. For an initial design, $25 \%$ is suggested for margin giving a total power of about 156W. This is somewhat consistent with the earlier estimate for a small spacecraft. The communications system can transmit at higher data rates with more power in an $\mathrm{L}_{2}$ halo orbit. Power estimates were given in Section 3.1 on communications and were from $50 \mathrm{~W}$ and up. The remainder of the spacecraft systems would still consume approximately the same power of about $100 \mathrm{~W}$, despite the increase in power for the communications system.

Solar cells are the primary power source. Using modern solar cells such as the multijunction GaInP/GaAs (Gallium Indium Phosphorous/Gallium Arsenide) to produce the power, efficiency in production of $22.0 \%$ can be achieved. Assuming a $15 \%$ loss in performance due to design and assembly, approximately $0.61 \mathrm{~m}^{2}$ of solar array are necessary to produce $156 \mathrm{~W}$ of power. For longer-term operation, solar arrays degrade at approximately $3 \%$ a year [16].

\subsection{Attitude Determination and Control}

The CRS requires an attitude determination and control system (ADCS) to determine the orientation of the spacecraft and place it in an orientation that is desirable to perform its functions [16]. The system also compensates for external disturbance torques such as solar pressure, gravitational forces brought upon by bodies other than the Earth and Moon, outgassing by materials on the spacecraft, and gravity gradient torques. The functions consist of pointing solar arrays at the Sun, aligning for burns of the main engine, and pointing its antennas at ground stations on Earth and at the lander in the Aitken Basin. This system contains devices for determining orientation such as Sun and star trackers and for controlling orientation such as momentum wheels and gas jets.

The main differences for the two mission orbits involve the antenna pointing requirements. In $\mathrm{L}_{2}$, the $\mathrm{CRS}$ traverses about a $6.3^{\circ}$ cone as viewed from the lander. The geometry is shown in Figure 21. As viewed from Earth, 
the halo orbit appears about the same size as the Moon's angular diameter of $0.5^{\circ}$. This traverse would be accomplished very slowly since the halo orbit period is approximately 10-20 days. This means the CRS does not need to track rapidly any objects. The potential exists to have very accurate pointing and thus maintain the data rates suggested for the CRS in an $\mathrm{L}_{2}$ halo orbit.

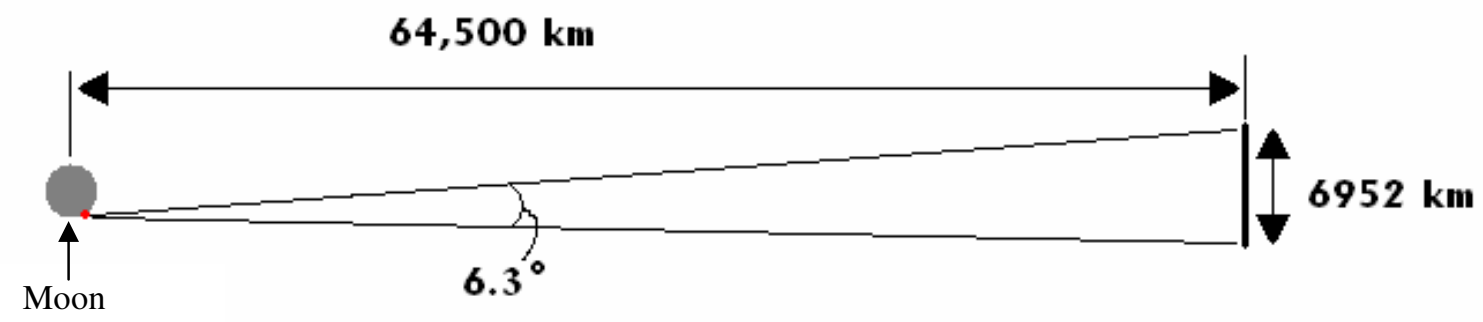

Figure 21 Communications Geometry for $L_{2}$ Halo Orbit

In LLPO, the CRS moves quickly across the sky relative to the lander requiring the antennas of the CRS and the lander to track each other to assure the high data rates discussed in Section 3.1 on communications. Since the data rates are very high, this generally means that the antenna beam will be narrow and it will have strict pointing requirements. This places a strain on the ADCS, but is achievable. The data rates could potentially be reduced in order to allow the CRS antennas to be pointed somewhat less accurately.

In addition to communication needs, the CRS needs to point its solar panels at the Sun to power itself and to align itself for burns of the main engine. Neither of these circumstances requires fast movement in LLPO or in $\mathrm{L}_{2}$.

With off-the-shelf hardware, attitude can be determined very accurately at a low cost, but to position the CRS accurately requires a greater cost in that knowledge of the CRS's center of mass and performance of the attitude control hardware must be known to a high degree of accuracy [16]. No particular design for this system can be disqualified, as any of the standard options for control such as cold gas jets, spin-stabilization, or 3-axis stabilization would meet the ADCS requirements. Gravity gradient stabilization is also possible, but this is probably not practical at $\mathrm{L}_{2}$. If the CRS is 3-axis stabilized, additional components are necessary for de-saturation of reaction or momentum wheels.

For an extended mission, efficiency is important, so dual spin-stabilization is desirable to reduce power consumption. Cold gas jets are not possible because carrying large amounts of gas would be voluminous and heavy. 


\subsection{Thermal/Environment}

The thermal and environmental system monitors and controls the temperatures of all the other systems and protects the spacecraft from the harsh effects of the space environment. Temperature sensors are required on all the major CRS components. These must be carefully monitored to ensure that the components stay within their desired operating temperatures. If the components near the limit of the desired temperatures, then a combination of louvers and heaters would be used to bring the components back within a safe operating range. Additional methods to regulate the component temperatures involve painting, insulating, the inclusion of radiation pellets and heat pipes, or a temporary reduction in data rate to reduce power usage [16].

The major thermal sources and sinks for the spacecraft are the Sun, internal heat, the Moon, and deep space that must be taken into account when designing the thermal system. The major difference between the two mission orbits is the amount of sunlight they receive. During the prime phase of the CRS's mission orbit in LLPO, due to the unique orientation of the orbit, solar eclipses are not frequent, especially at higher eccentricities. However, during an extended mission the CRS experiences solar eclipse more frequently. If the CRS is in an $\mathrm{L}_{2}$ halo orbit, it has continuous exposure during the mission time. For an extended mission, brief eclipses of the sun occur irregularly. The design of the thermal control would depend on the mission orbit. In LLPO, more heaters would be needed to keep the spacecraft components within their operating limits due to slightly greater number and length of eclipses. At $\mathrm{L}_{2}$, reorientation of the spacecraft on a regular basis is preferable so that the Sun's radiation would be distributed evenly on the spacecraft.

Throughout the CRS's lifetime, it is subjected to a variety of dangerous phenomena in the space environment [25]. The sun is the pre-dominant source of radiation; however, galactic particles can also affect the CRS. High energy protons and electrons are released via the solar wind and can either strike the spacecraft directly or be collected in the Van Allen belts through which the spacecraft must pass after launch from Earth [24]. For protection from solar radiation, various materials can be placed around the vital components absorbing the dangerous particles; this is known as radiation hardening. The electronics components also need to be designed to protect against single-event upsets. Other hazards such as meteoroids and space debris can impact a spacecraft at high relative velocities and damage components [25]. Shielding from these hazards is necessary and can be a dualfunction system designed to provide capability for both the thermal and the structural systems. For instance, 
aluminum shielding for protection from space debris can also act as a structural support and as a radiator for heat rejection.

\subsection{Command \& Data Handling}

The CRS contains a command and data handling $(\mathrm{C} \& \mathrm{DH})$ system to process commands received from ground stations on Earth for distribution to the spacecraft's systems or the lander, and to process data received from the spacecraft's systems or the lander for eventual transmittal to Earth [16]. The probable choice is a computer with some flight heritage to simplify the development and increase the reliability of the spacecraft. The typical system of Ref. 16 is assumed, as the demands on the computer are light. The system weighs from 4.5 to $6.5 \mathrm{~kg}$. It consumes approximately 13-18 W of power. Since the operation of both lander and CRS is contingent on the correct operation of command decoders on the CRS, careful design of the software systems is necessary. The CRS is commanding the lander and as such, it needs to maintain the integrity of commands. If commands issued by the CRS are misinterpreted by the lander, there is greater risk of mission failure due to the CRS intervening and possible time delays in ground control learning of the results of the commands.

\subsection{Structure}

The structure system provides mechanical support for all the other systems and provides an attachment point for the launch vehicle [16]. The structure likely supports multiple antennas and allows each antenna to be pointed independently. This requires antenna gimbals and significant volume on the structure. During launch, the spacecraft experiences extreme acceleration and vibration due to the effects of launch vehicle. This phase of the mission stresses the structure the greatest. The CRS therefore needs a strong structure to withstand these stresses, but it also must be lightweight and stiff. 


\section{Design}

The best spacecraft design is not necessarily the cheapest nor does the best mission design have the fastest transfer to the Moon. The best design comes from choosing the best plan that meets all the mission requirements. This consists of a reasonably quick transfer to lunar orbit. However, if the CRS mass reaches a point at which a slight reduction in mass enables a step-down in launch vehicle (and thus cost), then a slower transfer using less fuel is best chosen at a cost of increased time-of-flight. This means additional cost for engineers to be available to operate the spacecraft and more time for potential malfunctions on the CRS.

The mission requirements will dictate the choice of mission orbit. If the requirements are for maximum data return, an orbit that optimizes the CRS's viewing of the landing site such as a reasonably circular LLPO is the best option. Although it requires larger burns to enter orbit, has some eclipse time, and is limited in its viewing time of the lander it is attractive because of the significantly greater data rates. In the event that the lander lacks autonomy, the requirements may be for $100 \%$ viewing time. An orbit such as the $\mathrm{L}_{2}$ halo orbit is preferred. In addition, design engineers may need to work on Earth to troubleshoot a problem or scientists may need to hold meetings to decide what rocks to scoop and include in the sample return. More specific mission requirements will help to specify the importance of data return or viewing time. An $\mathrm{L}_{2}$ halo orbit or higher eccentricity LLPO cannot be completely ruled out. Even the lower limit of 2.7 gigabits for the $\mathrm{L}_{2}$ mission orbit is a significant amount of data return especially since it is over the lander continuously. If the lander could store some of the collected data onboard the lunar sample return capsule for recovery when the sample would arrive at Earth, this would lower the data transfer requirements further.

For the transfer orbit, a simple Hohmann type transfer is probably the best. This transfer has moderate $\Delta v$ costs, and a short TOF. Even though some orbits require significantly less $\Delta \mathrm{v}$, the TOF and the complicated engineering required to precisely design them argues against their use. In the event of a problem, such as a booster rocket failure whereby the CRS does not have enough velocity and fuel to reach the Moon, one possible recovery option is the use of one of the specialized, looping orbit trajectories as suggested by Refs.18 and 19.

As with any mission, the use of flight heritage is important in reducing costs and risks. Previously designed and flown hardware is preferred. For this mission, the CRS does not require any hardware that lacks flight heritage since many satellites have flown as communication relay satellites. However, the lander requires a specially designed system to collect a rock sample, protect it for a return to Earth via reentry through the atmosphere 
and landing on the surface. Thus, the majority of mission resources will likely be allocated to the task of designing the lander and not the CRS.

For the design of the CRS, the S-band communications system is recommended. A liquid propulsion system is suggested due to the high efficiency and ability to throttle the rocket. Power should be provided by solar cells, with batteries as a backup. The attitude should be determined with standard hardware such as Sun and star trackers and controlled using a system for 3-axis stabilization. 


\section{Mission Extension}

It is possible to configure the CRS for an extended mission. The CRS would remain in its mission orbit (LLPO or $\mathrm{L}_{2}$ halo orbit), available as a communications relay for future missions such as science, exploration or human missions. For example, it could be used for science missions that occur on the far side of the Moon. The Moon's far side would be a good place for astronomers to listen for electromagnetic emissions from outer space since there is no terrestrial interference. Data volume would be greater than it were for just the lunar sample return mission. The CRS could also be available for missions with astronauts whereby it simply acts as another communications method. It could be the initiation of a lunar communications system constellation.

There is a benefit for the communications system to be configured for high bandwidth usage since there may be a need to communicate in real-time with astronauts on the Moon. Other systems would see changes as well. For instance, since both batteries and solar cells tend to degrade over time, larger panels and greater amounts of batteries would be necessary to ensure production of the required power at end-of-life. The propulsion system would require additional station-keeping fuel to maintain the CRS's orbit and de-saturate the attitude control system. High efficiency of the attitude control system would be a requirement so a cold gas system would not be desirable, but a passive form of attitude control would be preferred. These changes would cause a domino effect on other systems, such as a larger structure to contain adequate volume for station-keeping fuel tanks and batteries. If the CRS was to be used for missions where a greater data volume would be transmitted, the onboard computer would need to have adequate memory for storing the data for later transmission to Earth. 


\section{Concluding Remarks}

A lunar sample return mission has the potential to return samples of the oldest rocks on the lunar surface. A communications relay satellite is needed to support the mission since the lander will not have line-of-sight communication with the Earth at the site where the oldest rocks are to be found, the Aitken Basin.

Mission orbits were examined for their capability to maximize data return to Earth and the total viewing time of the lander. These included low-lunar polar orbits, an $\mathrm{L}_{2}$ halo orbit, and lunar flybys. This was used in conjunction with a design for the CRS's communications system to approximate the data rates and total data return for a particular mission orbit. It was found that the LLPO mission types, for fixed communication components, can return much greater data, but at a cost of reduced viewing time when compared to the $\mathrm{L}_{2}$ halo orbit and flyby trajectory.

Transfer orbits were examined to estimate the required $\Delta \mathrm{v}$ and time-of-flight. Successive refinement of the transfer orbit was accomplished by including more factors that affect the trajectory. First, the simple two body case was used, then a patched conic, and last a non-coplanar trajectory. Reduction of $\Delta \mathrm{v}$ was possible, but at a cost of significantly increased flight time (several hundred days). Smaller reductions are possible, but for the first approximation of a spacecraft, these savings are likely to be within the design margins. Long TOF has several negative consequences, such as cost for spacecraft monitoring and increased risk of component failure, that make it unappealing unless a partial launch failure necessitates the use of a long TOF specialized trajectory to ensure mission success.

Several subsystems were examined for the different designs depending on the mission type. As discussed, the communications system power could be boosted to ensure higher data rates over longer distances. The power system required larger solar cells to generate enough power for the enhanced communications system. With the propulsion system design, significant fuel mass can be saved if spacecraft dry mass or $\Delta \mathrm{v}$ is reduced. ADCS would be designed with similar components, but its performance required different tracking capabilities due to the vast geometry differences among lunar orbit options. The other spacecraft systems remain largely the same for the main mission, but can change for the mission extension.

Areas of future work for this mission include understanding the science and data requirements of the lander. This is a high priority so the CRS can fulfill its role as communications relay for mission critical data. Some other possibilities include the study of the impact of having multiple landers collecting surface samples and how this 
affects the CRS. Estimates of cost and schedule must be developed and this mission provides a stepping stone for future Mars sample return missions as well as the first step in renewed lunar exploration for possible manned missions. 


\section{References}

1. Apollo 17. URL: http://nssdc.gsfc.nasa.gov/planetary/lunar/apollo17info.html [cited March 4, 2004].

2. NSSDC Master Catalogue: Spacecraft. Clementine, URL:

http://nssdc.gsfc.nasa.gov/database/MasterCatalog?sc=1994-004A [cited March 4, 2004].

3. NSSDC Master Catalogue: Spacecraft. Lunar Prospector, URL:

http://nssdc.gsfc.nasa.gov/database/MasterCatalog?sc=1998-001A [cited March 4, 2004].

4. NSSDC Master Catalogue: Spacecraft. Hiten, URL:

http://nssdc.gsfc.nasa.gov/database/MasterCatalog?sc=1990-007A [cited March 4, 2004].

5. NSSDC Master Catalogue: Spacecraft. SMART-1, URL:

http://nssdc.gsfc.nasa.gov/database/MasterCatalog?sc=2003-043C [cited March 4, 2004].

6. Soviet Missions to the Moon. URL: http://nssdc.gsfc.nasa.gov/planetary/lunar/lunarussr.html [cited March 4, 2004].

7. Space Studies Board, Division on Engineering and Physical Sciences, National Research Council of the National Academies, New Frontiers in the Solar System: An Integrated Exploration Strategy, Solar System Exploration Survey, The National Academies Press, Washington, DC, 2003.

8. New Frontiers. Office of Space Sciences, NASA, URL:

http://research.hq.nasa.gov/code_s/nra/current/AO-03-OSS-03/main.html [cited February, 27 2004]

9. Clementine images from the Moon. URL: http://www.lpi.usra.edu/research/clemen/clemen.html [cited March 4, 2004].

10. Young, Thomas et al. "Mars Program Independent Assessment Team Report.” NASA-00139, Washington, D.C.: National Aeronautics and Space Administration, March 14, 2000. URL: http://www.jpl.nasa.gov/marsreports/mpiat_report.pdf [cited March 23, 2004].

11. Mars Exploration: Missions. NASA, URL: http://marsprogram.jpl.nasa.gov/missions/log/ [cited March 15, 2004].

12. Vallado, David A. Fundamentals of Astrodynamics and Applications, $2^{\text {nd }}$ ed., El Segundo, CA: Microcosm Press, Dordrecht, Netherlands: Kluwer Academic Publishers, 2001.

13. Farquhar, Robert W. "The Utilization of Halo Orbits in Advanced Lunar Operations." NASA TN D-6365, 1971. 
14. Corliss, Jim. Personal Communications, September 2003.

15. STK, Satellite Tool Kit, Software Package Ver. 5.0, Analytical Graphics Inc., Malvern, PA, 2003.

16. Wertz, James R. and Wiley J. Larson, (eds.), Space Mission Analysis and Design. $3^{\text {rd }}$ ed. El Segundo, CA: Microcosm Press, Dordrecht, Netherlands: Kluwer Academic Publishers, 1999.

17. Bate, Roger R., Donald D. Mueller, and Jerry E. White. Fundamentals of Astrodynamics. New York: Dover Publications, Inc., 1971.

18. Bailey, Stephen A. et al. “Common Lunar Lander.” AIAA 92-1481.

19. Krish, V., E.A. Belbruno, W.M. Hollister. “An Investigation into Critical Aspects of a New Form of Low Energy Lunar Transfer, The Belbruno-Miller Trajectories.” AIAA 92-4581-CP.

20. Lo, Martin W., Min-Kin J. Chung. "Lunar Sample Return via the Interplanetary Superhighway.” AIAA 2002-4718.

21. Apollo 11 Press Kit. NASA Release No: 69-83K. July 6, 1969, URL: http://wwwlib.ksc.nasa.gov/lib/archives/apollo/pk/APOLLO11pt1.PDF [cited 26 May 2004].

22. Apollo 17 Press Kit. NASA Release No: 72-220K. November 26, 1972, URL: http://wwwlib.ksc.nasa.gov/lib/archives/apollo/pk/1APOLLO17.PDF [cited 26 May 2004].

23. Physical ephemeris for the solar system objects (IMC) http://www.imcce.fr/ephem/ephephys_eng/ephephys_f1.html [cited March 4, 2004].

24. Kowitz, Herb. Personal Communication, May-August 2003.

25. Fortescue, Peter and John Stark, (eds.), Spacecraft Systems Engineering. $2^{\text {nd }}$ ed. New York: John Wiley and Sons, 1995. 


\section{Appendix A-Text of the New Frontiers Announcement of Opportunity Describing the Lunar Mission Science Requirements}

\subsubsection{Lunar South Pole-Aitken Basin Sample Return}

The surface of the South Pole-Aitken basin, located on the Moon's far side southern polar region, is likely to contain some fraction of the mineralogy of the Moon's lower crust. Samples of these ancient materials that are not biased by nearside impact basin formation are highly desirable to further understand the history of Earth's Moon. Therefore, a mission to return a sufficient sample of material from the heretofore-unsampled South Pole-Aitken basin terrain, including useful samples from the deep crust of the early Moon, should accomplish (following chemical, isotopic, and petrologic analysis of returned materials as well as radiometric age dating on Earth) the majority of following science objectives:

- Elucidate the nature of the Moon's lower crust and mantle by direct measurements of its composition and of sample ages;

- Determine the chronology of basin-forming impacts and constrain the period of late, heavy bombardment in the inner solar system, and thus, address fundamental questions of inner solar system impact processes and chronology;

- Characterize a large lunar impact basin through "ground truth" validation of global, regional, and local remotely sensed data of the sampled site;

- Elucidate the sources of thorium and other heat-producing elements in order to understand lunar differentiation and thermal evolution; and

- Determine ages and compositions of far-side basalts to determine how mantle source regions on the far side of the Moon differ from regions sampled by Apollo and Luna basalts

The Decadal Survey description of this mission places very high priority on the return of useful samples from the deep crust of the early Moon. The strawman mission considered by the Decadal Study is a robotic lander with automatic scooping and sieving capability to enhance the return of rock fragments and also to provide context to sampled areas. The return of at least $1 \mathrm{~kg}$ of sampled materials is expected. However, any mission architecture that returns a sample assemblage adequate to achieve the majority of the science objectives stated above for a cost within the New Frontiers cost cap will be considered responsive to this AO.

http://research.hq.nasa.gov/code_s/nra/current/AO-03-OSS-03/main.html\#2.1.3 


\section{Appendix B-Computer Codes (Matlab)}

This calculates the non-coplanar lunar trajectory utilizing noncoplanar_lunar_traj.m, free_flight_angle.m, LSTtime.m, JDtoGregorianDate.m, and neu_to_anomaly.m It outputs the date and time of launch, launch azimuth and several other parameters given the desired time of intercept and several other flight parameters.

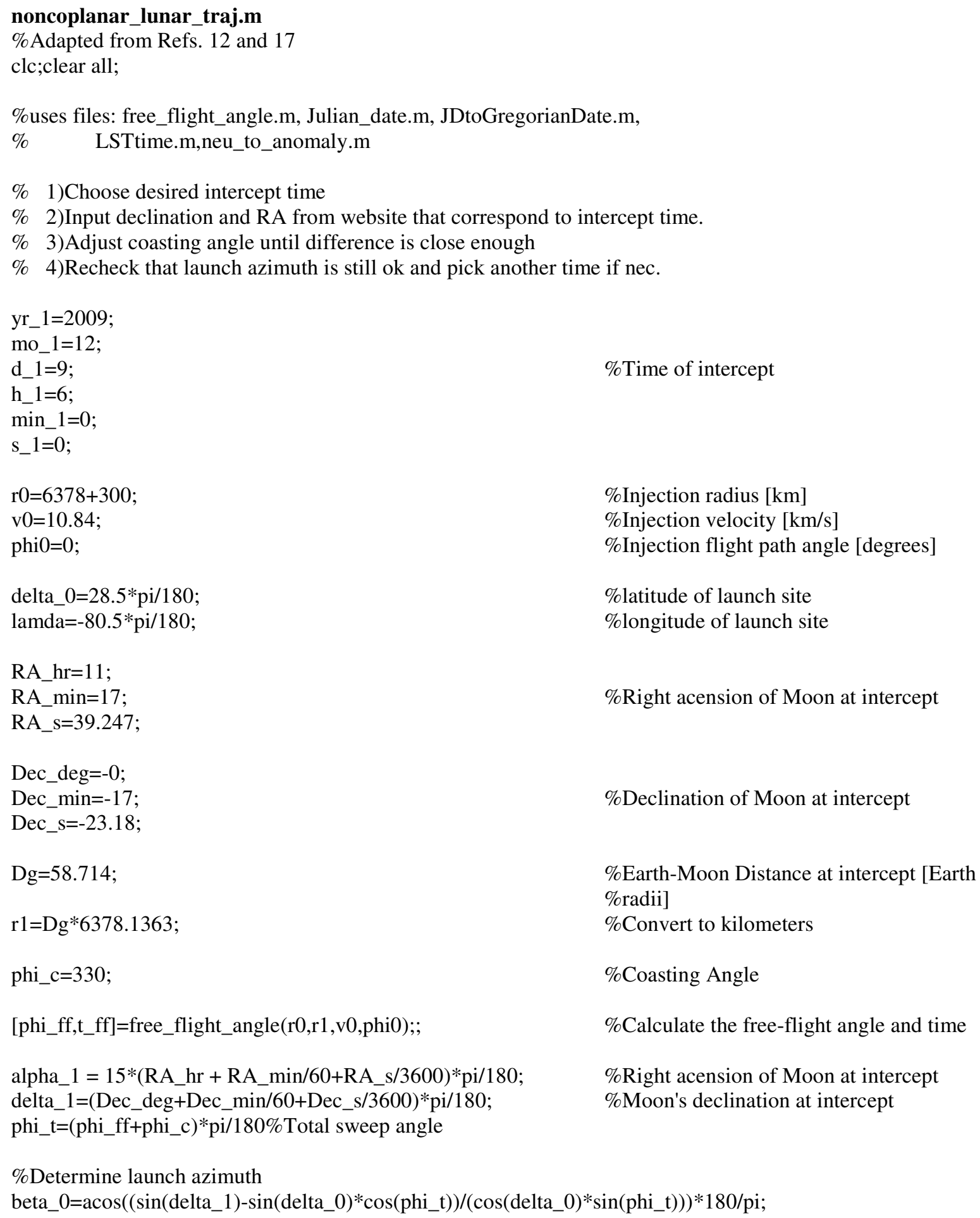

\%Right acension of Moon at intercept

$\%$ Declination of Moon at intercept

\%Earth-Moon Distance at intercept [Earth \%radii]

$\%$ Convert to kilometers

\%Coasting Angle

$\%$ Calculate the free-flight angle and time

\%Right acension of Moon at intercept $\%$ Moon's declination at intercept 
neu $0=0 *$ pi $/ 180$;

neu1=phi_c*pi/180;

$\mathrm{mu}=398600.4415$;

$\mathrm{e}=0$;

$\mathrm{a}=6678.1363$

[E0]=neu_to_anomaly(e,neu0*180/pi);

$\%$ Determine coasting time

[E1]=neu_to_anomaly(e,neu $1 * 180 / \mathrm{pi})$;

tof $1=\operatorname{sqrt}\left(\mathrm{a}^{\wedge} 3 / \mathrm{mu}\right) *\left(\left(\mathrm{E} 1-\mathrm{e}^{*} \sin (\mathrm{E} 1)\right)-\left(\mathrm{E} 0-\mathrm{e}^{*} \sin (\mathrm{E} 0)\right)\right)$;

t_c=tof $1 / 3600 / 24$;

$\mathrm{t} \_\mathrm{t}=\mathrm{t} \_\mathrm{ff}+\mathrm{t} \_\mathrm{c}$

$\%$ Total time from launch to intercept

[t_1]=Julian_date $\left(\mathrm{yr} \_1, \mathrm{mo} \_1, \mathrm{~d} \_1, \mathrm{~h} \_1, \mathrm{~min} \_1, \mathrm{~s} \_1\right)$;

$\mathrm{t} \_0=\mathrm{t} \_1-\mathrm{t} \_\mathrm{t}$

$\%$ Determine launch time

[date0]=JDtoGregorianDate(t_0);

[year_0,Mon_0,day_0,h_0,min_0,s_0]=datevec(date0);

[GMST,alpha_0]=LSTtime(year_0,Mon_0,day_0,h_0,min_0,s_0,lamda);

alpha_0=mod(alpha_0,360);

RA_minus_LST $=$ mod(alpha_1 $* 180 /$ pi-alpha_0,360);

d_alpha $=\operatorname{acos}\left((\cos (\right.$ phi_t $)-\sin ($ delta_0 $) * \sin ($ delta_1 $)) /\left(\cos \left(\operatorname{delta} \_0\right) * \cos (\right.$ delta_1 $\left.\left.)\right)\right) * 180 /$ pi;

temp=alpha_0/15;

alpha_0_hr=floor(temp);

alpha_0_min=floor((temp-alpha_0_hr)*60);

alpha_0_s=(temp-alpha_0_hr-alpha_0_min/60)*3600;

$\%$ Several lines of code for easy-to-read display of output

disp('Launch parameters')

disp('Date and time')

$\operatorname{disp}($ date 0$)$

$\operatorname{disp}('$ ' )

disp('Launch Azimuth (acceptable is 40->115)')

disp(beta_0)

disp('Right Ascension of the Launch site (hr min s)')

disp([alpha_0_hr,alpha_0_min,alpha_0_s])

disp('Launch and coasting angle (degrees) and time (days)')

disp([phi_ct_c])

disp('Intercept parameters')

disp('Date and time')

date1=datestr(datenum(yr_1,mo_1,d_1,h_1,min_1,s_1),21);

disp(date1)

$\operatorname{disp}('$ ' )

disp('RA of Moon at intercept (hr min s)')

disp([RA_hr,RA_min,RA_s])

$\operatorname{disp}($ 'Declination of Moon at intercept(deg min s)')

disp([Dec_deg,Dec_min,Dec_s] $)$

disp('Free flight angle (degrees) and time (days)')

disp([phi_ff t_ff] $)$

disp('Difference in right acension between launch and intercept')

disp(d_alpha) 
disp('delta_alpha - (alpha_1 - alpha_0)')

disp('Should be less than absolute value of one degree')

disp(d_alpha-RA_minus_LST)

This generates the free-flight angle and time of free flight based on flight parameters similar to the discussion on p.328 of Ref.17.

\section{free_flight_angle.m}

function [phi_ff,t_ff] $=$ free_flight_angle(r0,r1,v0,phi0)

$\%$

$\%$

$\%$

$\%$

$\%$

$\mathrm{mu}=398600.4415$

Eng=v0.^2/2-mu/r0;

$\mathrm{a}=-\mathrm{mu} . /(2 * \mathrm{Eng})$;

$\mathrm{h}=\mathrm{r} 0 * \mathrm{v} 0 * \cos (\mathrm{phi} 0 * \mathrm{pi} / 180)$;

$\mathrm{p}=\mathrm{h}^{\wedge} 2 / \mathrm{mu}$

$\mathrm{e}=\operatorname{sqrt}(1-\mathrm{p} / \mathrm{a})$;

neu $1=\operatorname{acos}((\mathrm{p}-\mathrm{r} 1) /(\mathrm{e} * \mathrm{r} 1))$;

neu $0=\operatorname{acos}((\mathrm{p}-\mathrm{r} 0) /(\mathrm{e} * \mathrm{r} 0))$;

[E0]=neu_to_anomaly(e,neu $0 * 180 / \mathrm{pi})$;

[E1]=neu_to_anomaly $(\mathrm{e}, \mathrm{neu} 1 * 180 / \mathrm{pi})$;

tof $1=\operatorname{sqrt}\left(\mathrm{a}^{\wedge} 3 / \mathrm{mu}\right) *\left(\left(\mathrm{E} 1-\mathrm{e}^{*} \sin (\mathrm{E} 1)\right)-\left(\mathrm{E} 0-\mathrm{e}^{*} \sin (\mathrm{E} 0)\right)\right)$;

t_ff=tof $1 / 3600 / 24$;

phi_ff=real(neu $1 * 180 /$ pi-neu $0 * 180 /$ pi); [phi_ff,t_ff]=free_flight_angle.m(r0,r1,v0,fpa) $\mathrm{r} 0$ is the injection altitude above the Earth [km] $\mathrm{r} 1$ is the orbital radius of the Moon [km] $\mathrm{v} 0$ is the injection velocity $[\mathrm{km} / \mathrm{s}]$ phi0 is the flight path angle at injection [degrees]

$\%$ Gravitational parameter for Earth $\%\left[\mathrm{~km}^{\wedge} 3 / \mathrm{s}^{\wedge} 2\right]$

$\%$ Orbital energy $\left[\mathrm{km}^{\wedge} 2 / \mathrm{s}^{\wedge} 2\right]$

$\%$ Semi-major axis $[\mathrm{km}]$

$\%$ Calculate angular momentum

$\%$ Calculate parameter

$\%$ Calculate eccentricity

This generates the Greenwich Mean Sidereal time and local sidereal time from an input of a date, time and longitude.

\section{LSTtime.m}

function [GMST,LST]=LSTtime(yr,mo,d,h,min,s,lamda)

$\% \quad$ [GMST,LST] $=\mathrm{LSTtime}(\mathrm{yr}, \mathrm{mo}, \mathrm{d}, \mathrm{h}, \mathrm{min}, \mathrm{s}, \mathrm{lamda})$

$\%$

$\%$ Generates the GMST and LST for a give date, time, and position where GMST

$\%$ is the Greenwich Mean Sidereal Time in degrees, LST is the Local Sidereal Time

$\%$ Input are the year (yr), month (mo) (A number from 1 to 12), day (A

$\%$ number from 1 to 29-31 depending on month), hour of day (h), minute

$\%$ of hour (min), and second (s). This must be in terms of UTC. The last

$\%$ input (lamda) is the longitude which varies from -180 to 180 with positive

$\%$ values being east and negative being west.

$[\mathrm{m}, \mathrm{n}]=$ size $($ lamda);

if lamda $>180$ llamda $<-180 \mid(m+n) \sim=2$

error('Input longitude must be a scalar value between -180 and 180')

end

[JD]=Julian_date(yr,mo,d,h,min,s); 
Tut1=(JD-2451545)/36525;

GMST_sec $=67310.54841+(876600 * 3600+8640184.812866) *$ Tut $1+.093104 * T_{u t 1}{ }^{\wedge} 2-6.2 \mathrm{e}-6 * \mathrm{Tut}^{\wedge}{ }^{\wedge} 3$;

GMST $=360+$ rem(GMST_sec, 86400$) / 240$;

$\mathrm{LST}=\mathrm{GMST}+$ lamda;

This transforms an input date in Julian Days to a more conventional looking date.

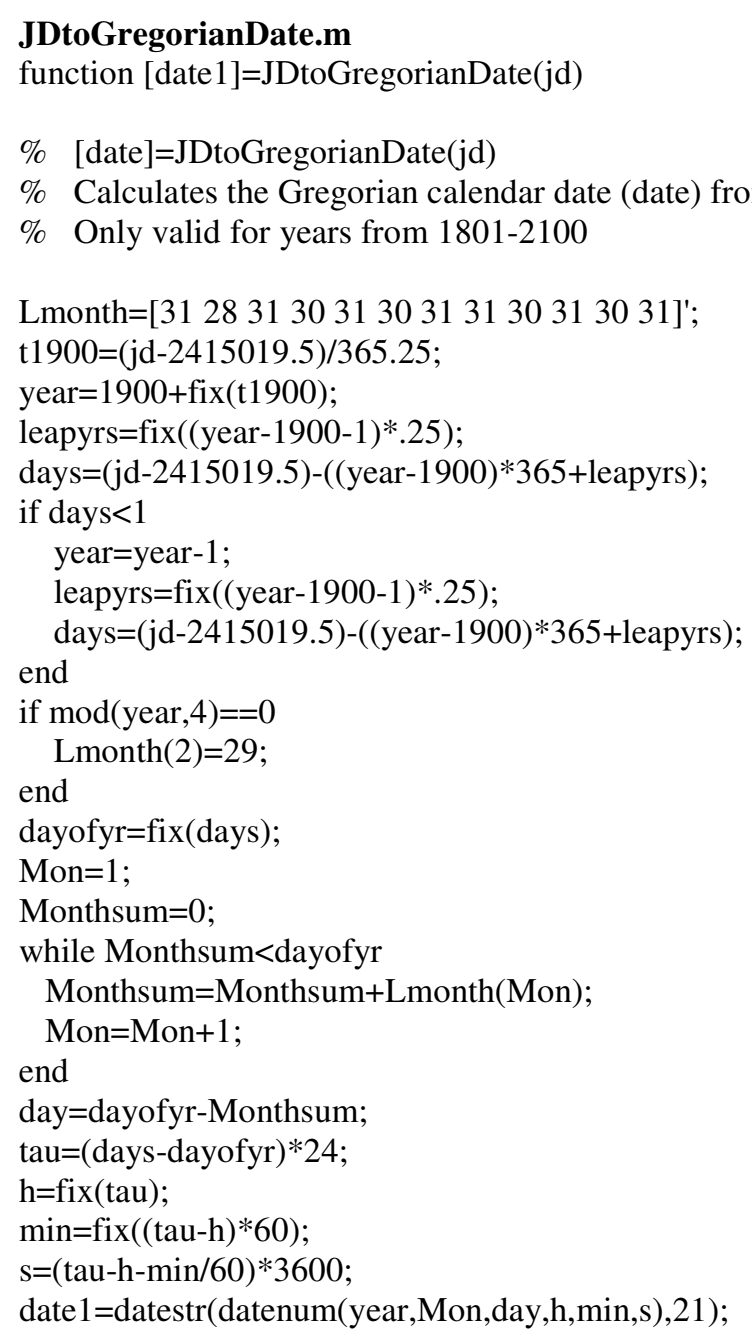

This transforms the true anomaly into the eccentric, hyperbolic or parabolic anomaly depending on the input eccentricity.

\section{neu_to_anomaly.m}

function [anomaly]=neu_to_anomaly(e,neu)

$\%$

$\%$ [anomaly]=neu_to_anomaly(e,neu) Input is the e (eccentricity) and neu (the

$\%$ true anomaly, in degrees). Output is the appropriate anomaly for the type of

$\%$ orbit in units of radians.

neu=neu*pi/180;

if $\mathrm{e}<1$

anomalytemp $=\operatorname{atan} 2\left(\sin (n e u) * \operatorname{sqrt}\left(1-\mathrm{e}^{\wedge} 2\right) /\left(1+\mathrm{e}^{*} \cos (\mathrm{neu})\right),(\mathrm{e}+\cos (\mathrm{neu})) /\left(1+\mathrm{e}^{*} \cos (\mathrm{neu})\right)\right)$;

if anomalytemp $<0$ 


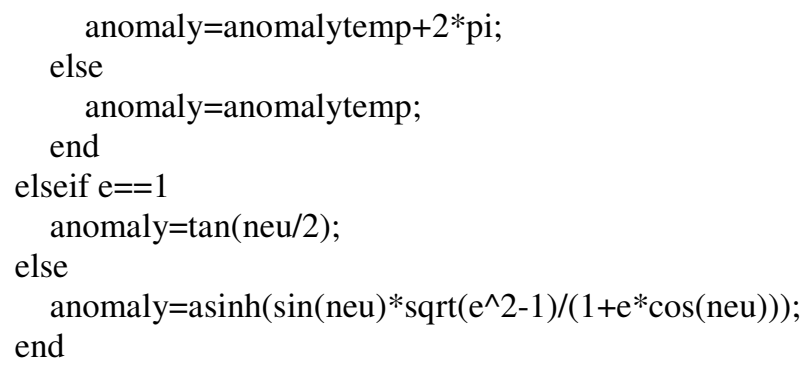

This calculates a patched conic trajectory and is based on discussion in the paper.

\section{patchedconic.m}

function [close_height,TOF_days,rp,vp,e2,gamma0]=patchedconic(r0,v0,phi0,lamda1,D) $\%$ Adapted from Ref 17

$\%$

$\% \mathrm{r} 0$ is the initial orbital radius around the earth in $\mathrm{km}$.

$\% \mathrm{v} 0$ is the initial orbital velocity once the "kick" has been applied in $\mathrm{km} / \mathrm{s}$.

$\%$ phi0 is the flight path angle at departure in radians.

$\%$ lamda1 specifies the point at which the geocentric trajectory crosses the

$\%$ lunar sphere of influence in radians.

$\% \mathrm{D}$ is lunar orbital radius in $\mathrm{km}$.

$\mathrm{mu}=398600.441$;

mum=4902.799;

$w m=2.649 \mathrm{e}-6$;

$\mathrm{Rs}=\mathrm{D} *(\mathrm{mum} / \mathrm{mu})^{\wedge}(2 / 5)$;

$\mathrm{E}=\mathrm{v} 0^{\wedge} 2 / 2-\mathrm{mu} / \mathrm{r} 0$

$\mathrm{h}=\mathrm{r} 0 * \mathrm{v} 0 * \cos (\mathrm{phi} 0)$;

$\mathrm{r} 1=\operatorname{sqrt}\left(\mathrm{D}^{\wedge} 2+\mathrm{Rs}^{\wedge} 2-2 * \mathrm{D} * \mathrm{Rs}^{*} \cos (\right.$ lamda 1$\left.)\right)$;

$\mathrm{v} 1=\operatorname{sqrt}(2 *(\mathrm{E}+\mathrm{mu} / \mathrm{r} 1))$;

if $\operatorname{abs}(\operatorname{imag}(\mathrm{v} 1))>1 \mathrm{e}-7$

warning('Orbit not energtic enough to reach Moon SOI')

end

phil $=\operatorname{acos}(\mathrm{h} / \mathrm{r} 1 / \mathrm{v} 1)$;

$\operatorname{gamma} 1=\operatorname{asin}(\mathrm{Rs} / \mathrm{r} 1 * \sin ($ lamda 1$))$;

$\mathrm{p}=\mathrm{h}^{\wedge} 2 / \mathrm{mu}$

$\mathrm{a}=-\mathrm{mu} / 2 / \mathrm{E}$;

$\mathrm{e}=\operatorname{sqrt}(1-\mathrm{p} / \mathrm{a})$;

neu $0=\operatorname{acos}((\mathrm{p}-\mathrm{r} 0) /(\mathrm{r} 0 * \mathrm{e}))$;

neu $1=\operatorname{acos}((\mathrm{p}-\mathrm{r} 1) /(\mathrm{r} 1 * \mathrm{e}))$;

$\mathrm{E} 0=\operatorname{acos}\left((\mathrm{e}+\cos (\right.$ neu 0$)) /\left(1+\mathrm{e}^{*} \cos (\right.$ neu 0$\left.\left.)\right)\right)$;

$\mathrm{E} 1=\operatorname{acos}\left((\mathrm{e}+\cos (\right.$ neu 1$)) /\left(1+\mathrm{e}^{*} \cos (\right.$ neu 1$\left.\left.)\right)\right)$;

tof $1=\operatorname{sqrt}\left(\mathrm{a}^{\wedge} 3 / \mathrm{mu}\right) *\left(\left(\mathrm{E} 1-\mathrm{e}^{*} \sin (\mathrm{E} 1)\right)-\left(\mathrm{E} 0-\mathrm{e}^{*} \sin (\mathrm{E} 0)\right)\right) / 3600 / 24$;

gamma0 $=($ neu1-neu0-gamma1-wm*tof1 $) * 180 /$ pi;

if imag $($ gamma0 $)>0$

warning('Phase angle is imaginery')

end

$\%$ Conditions at patch point

r2=Rs;

$\mathrm{vm}=1.018$; 
$\mathrm{v} 2=\operatorname{sqrt}\left(\mathrm{v} 1^{\wedge} 2+\mathrm{vm}^{\wedge} 2-2 * \mathrm{v} 1 * \mathrm{vm} * \cos (\right.$ phi1-gamma 1$\left.)\right)$;

epilson $2=\operatorname{asin}(\mathrm{vm} / \mathrm{v} 2 * \cos ($ lamda 1$)-\mathrm{v} 1 / \mathrm{v} 2 * \cos ($ lamda $1+$ gamma $1-$ phi 1$))$;

$\mathrm{E} 2=\mathrm{v} 2 \wedge 2 / 2-\mathrm{mum} / \mathrm{r} 2$

$\mathrm{h} 2=\mathrm{r} 2 * \mathrm{v} 2 * \sin ($ epilson 2$)$;

$\mathrm{p} 2=\mathrm{h} 2^{\wedge} 2 / \mathrm{mum}$;

$\mathrm{e} 2=\operatorname{sqrt}\left(1+2 * \mathrm{E} 2 * \mathrm{~h} 2 \wedge 2 / \mathrm{mum}^{\wedge} 2\right)$;

$\mathrm{rp}=\mathrm{p} 2 /(1+\mathrm{e} 2)$;

$\mathrm{vp}=\operatorname{sqrt}(2 *(\mathrm{E} 2+\mathrm{mum} / \mathrm{rp}))$;

$\mathrm{Rm}=1738$;

close_height $=\mathrm{rp}-\mathrm{Rm}$;

if close_height $<0$

warning('Spacecraft collides with Moon')

end

$\%$ Calculations to find TOF inside lunar SOI

a2=-mum $/(2 * \mathrm{E} 2)$;

neu2 $=\operatorname{acos}((\mathrm{p} 2-\mathrm{r} 2) /(\mathrm{r} 2 * \mathrm{e} 2))$;

neu3 $=\operatorname{acos}((\mathrm{p} 2-\mathrm{rp}) /(\mathrm{rp} * \mathrm{e} 2))$;

$\mathrm{E} 2=\operatorname{acos}((\mathrm{e} 2+\cos ($ neu 2$)) /(1+\mathrm{e} 2 * \cos ($ neu 2$)))$;

$\mathrm{E} 3=\operatorname{acos}((\mathrm{e} 2+\cos ($ neu3 $)) /(1+\mathrm{e} 2 * \cos ($ neu 3$)))$;

tof $2=\operatorname{sqrt}\left(\mathrm{a} 2{ }^{\wedge} 3 / \mathrm{mum}\right) *((\mathrm{E} 3-\mathrm{e} 2 * \sin (\mathrm{E} 3))-(\mathrm{E} 2-\mathrm{e} 2 * \sin (\mathrm{E} 2))) / 3600 / 24$;

TOF_days $=($ tof $1+$ tof 2$)$;

This utilizes the function file patched_conic.m.

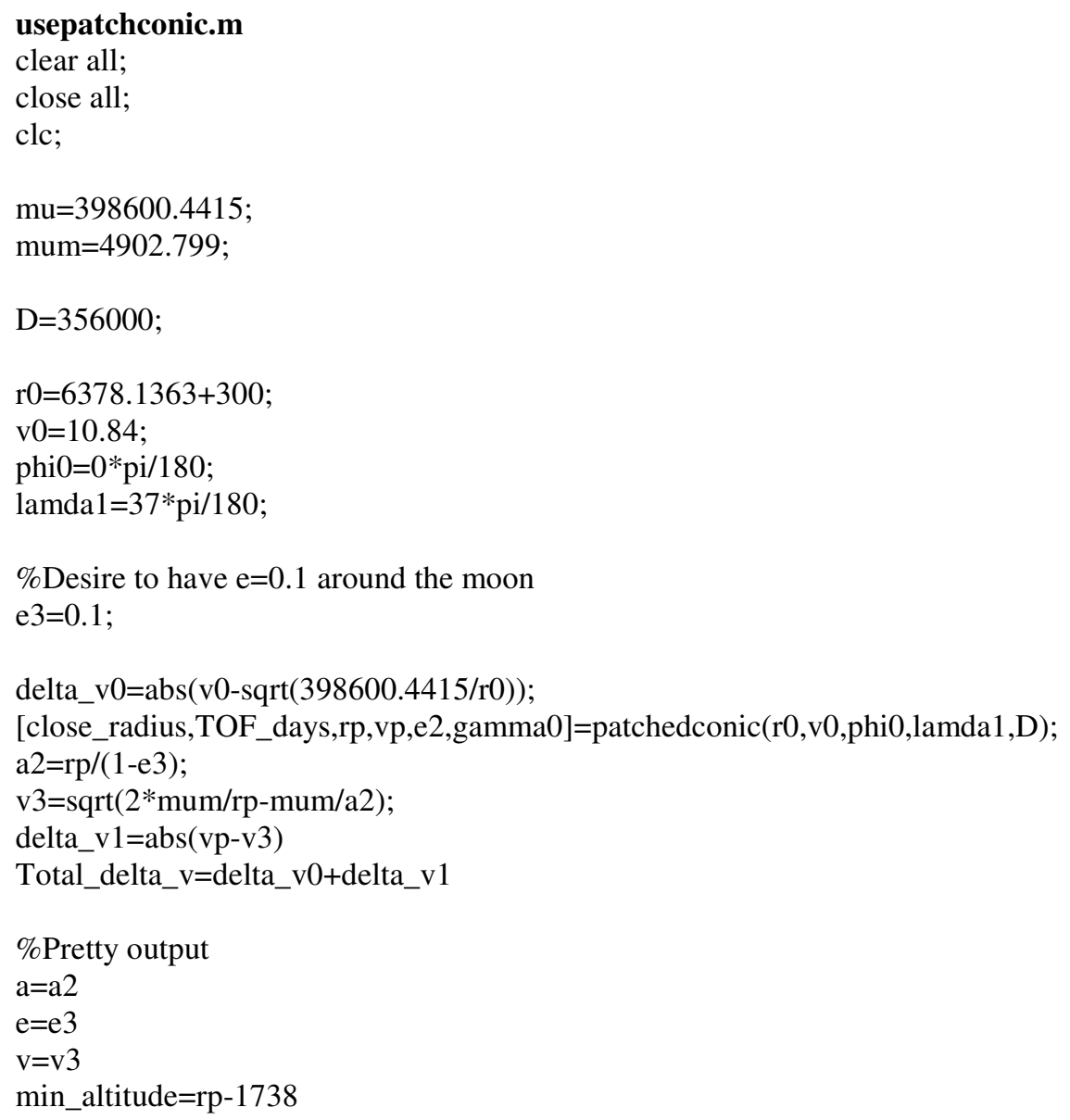


max_altitide $=\mathrm{a} *(1+\mathrm{e})-1738$

TOF_days=TOF_days 


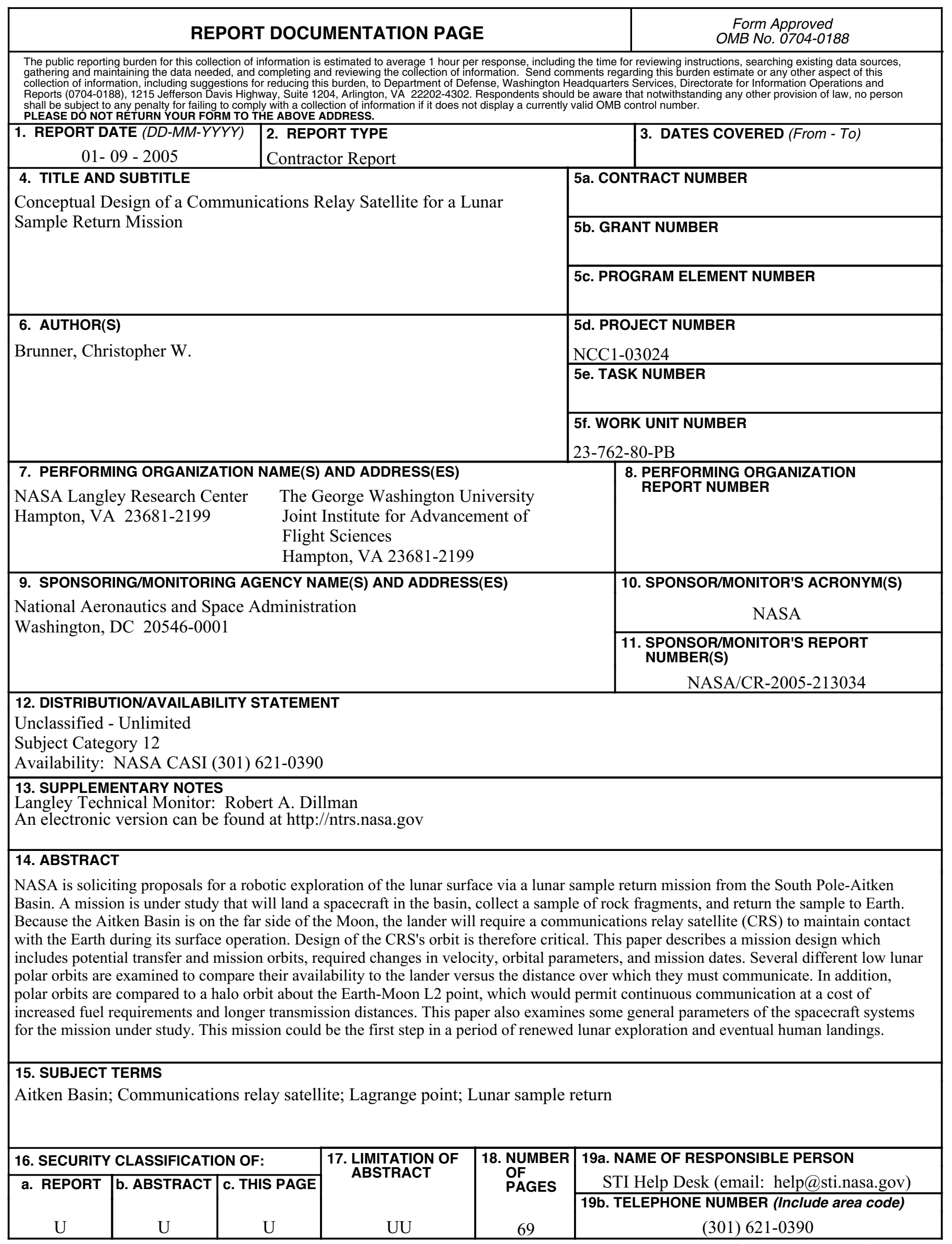

